\title{
ARTICLE
}

\section{A vaccine combination of lipid nanoparticles and a cholera toxin adjuvant derivative greatly improves lung protection against influenza virus infection}

\author{
Valentina Bernasconi ${ }^{1}$, Karin Norling ${ }^{2}$, Inta Gribonika ${ }^{1}$, Li Ching Ong ${ }^{1}$, Sabina Burazerovic ${ }^{2}$, Nagma Parveen ${ }^{2}$, Karin Schön ${ }^{1}$, \\ Anneli Stensson ${ }^{1}$, Marta Bally ${ }^{3}$, Göran Larson ${ }^{4}$, Fredrik Höök ${ }^{2}$ and Nils Lycke ${ }^{1}$
}

This is a proof-of-principle study demonstrating that the combination of a cholera toxin derived adjuvant, CTA1-DD, and lipid nanoparticles (LNP) can significantly improve the immunogenicity and protective capacity of an intranasal vaccine. We explored the self-adjuvanted universal influenza vaccine candidate, CTA1-3M2e-DD (FPM2e), linked to LNPs. We found that the combined vector greatly enhanced survival against a highly virulent PR8 strain of influenza virus as compared to when mice were immunized with FPM2e alone. The combined vaccine vector enhanced early endosomal processing and peptide presentation in dendritic cells and upregulated co-stimulation. The augmenting effect was CTA1-enzyme dependent. Whereas systemic anti-M2e antibody and CD4 T-cell responses were comparable to those of the soluble protein, the local respiratory tract IgA and the specific Th1 and Th17 responses were strongly enhanced. Surprisingly, the lung tissue did not exhibit gross pathology upon recovery from infection and M2e-specific lung resident $\mathrm{CD}^{+}{ }^{+} \mathrm{T}$ cells were threefold higher than in FPM2e-immunized mice. This study conveys optimism as to the protective ability of a combination vaccine based on LNPs and various forms of the CTA1-DD adjuvant platform, in general, and, more specifically, an important way forward to develop a universal vaccine against influenza.

Mucosal Immunology (2021) 14:523-536; https://doi.org/10.1038/s41385-020-0334-2

\section{INTRODUCTION}

Despite that mucosal vaccines have been found more effective than injectable vaccines for stimulating local protection in the respiratory and gastrointestinal tracts, only a handful of such commercial vaccines exist today. ${ }^{1}$ This is partly because of a lack of effective, yet nontoxic and safe, adjuvants that can enhance immune responses against the vaccine antigens. ${ }^{2}$ An additional problem is that mucosal vaccines may need protection against proteolytic degradation, and whereas this has been found easily achieved by packaging vaccine antigens into nanoparticles, such particulate vaccines have often been found insufficiently immunogenic. $^{3}$ Therefore, a combination of the two strategies appears attractive, but few studies have reported on such successful combinations. $^{2}$ The physical linking of antigen and adjuvant in a nanoparticle would have obvious benefits with regard to dose sparing and targeting to relevant tissues and cells, which would also dramatically reduce potential side effects. ${ }^{4}$ However, most studies have investigated the combination of toll-like receptor ligands such as CpG or flagellin with nanoparticles resulting in an increased risk for inflammatory responses and other unwanted side effects. ${ }^{5}$

There are several merits of mucosal vaccines, the most important being that these, as opposed to injectable vaccines, effectively stimulate tissue resident memory $\mathrm{CD}^{+}$and $\mathrm{CD}^{+}$
T cells and local IgA antibody production. ${ }^{6}$ Indeed, intranasal immunization (i.n.) stimulates both strong local respiratory tract immunity and significant serum IgG antibody responses. ${ }^{7}$ Moreover, mucosal vaccines are needle free and have better safety, compliance, and ease of administration compared to injectable vaccines. $^{8}$ In case of mass vaccination, due to epidemic or pandemic spread of infection, mucosal vaccines are superior to injected vaccines and significantly lower the risk of transmitting infections. ${ }^{9}$ The use of lipid nanoparticles (LNP) for vaccine delivery has proven efficient for mucosal immunization, in particular for intranasal immunizations. ${ }^{10}$ Liposomes are clinically approved for drug delivery and have also been extensively studied as vehicles for vaccine antigens as they provide highly versatile and flexible systems. ${ }^{1,2}$ These carriers are spherical lipid bilayer structures with an aqueous core ranging in size from tens of nanometers to several micrometers in diameter. ${ }^{11}$ Hydrophobic peptides or proteins can be inserted into the inner hydrophobic center of the bilayer, while hydrophilic molecules can either be encapsulated in the core or bound to the surface of the LNPs. In this way, antigen degradation can be prevented and the uptake and processing of antigen by antigen-presenting cells (APC) facilitated. ${ }^{12}$ Importantly, the physicochemical properties of the LNPs are highly adaptable and their size, charge, and lamellarity can easily be tailored for distinct purposes. ${ }^{2}$ Hence, many

\footnotetext{
${ }^{1}$ Department of Microbiology and Immunology, Institute of Biomedicine, Mucosal Immunobiology and Vaccine Center (MIVAC), University of Gothenburg, 405 30 Gothenburg, Sweden; ${ }^{2}$ Division of Biological Physics, Department of Physics, Chalmers University of Technology, 41296 Gothenburg, Sweden; ${ }^{3}$ Department of Clinical Microbiology and

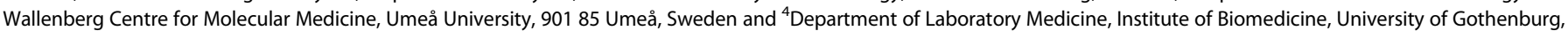
41345 Gothenburg, Sweden

Correspondence: Nils Lycke (nils.lycke@microbio.gu.se)
}

Received: 6 November 2019 Revised: 20 July 2020 Accepted: 24 July 2020

Published online: 17 August 2020 
modifications can be made to the LNPs that further improve and adapt their function to the needs of mucosal vaccines. The LNPs can also be equipped with cell-targeting molecules such as dendritic cell (DC)-specific antibodies, of which DEC-205 is one example, or with polymers, such as polyethylene glycol (PEG), for increasing the protein binding capacity or the retention time of nanoparticles in the circulation. However, only few previous reports have attempted to combine an already effective mucosal adjuvant with LNPs. ${ }^{2}$ We have developed the CTA1-DD adjuvant to circumvent the toxicity of the cholera toxin (CT) molecule, which, otherwise, is considered the gold standard for mucosal adjuvants. ${ }^{13}$ Whereas the B-subunits from the CT holotoxin were eliminated and replaced with a dimer of fragment $D$ from Staphylococcus aureus protein A, we were also able to eliminate the toxic side effects with retained full adjuvant activity following i.n. administration. ${ }^{14}$ The mechanism for the adjuvant effect is harnessed in the ADP-ribosylating activity of the CTA1-enzyme, which is known to activate the targeted cell via Gsa at the cell membrane. ${ }^{15}$ Mutants with an inactive enzyme, such as the CTA1 (R9K)-DD molecule, have lost their immunoenhancing function. ${ }^{16}$ It is known from recent studies that CTA1-DD targets DCs after i.n. administration and these cells are the critical cells to prime T-cell responses in the draining mediastinal lymph node $(\mathrm{mLN})$ following immunization. ${ }^{13}$ Similar to CT holotoxin the admixing of CTA1-DD to vaccine antigens strongly augments humoral as well as cellmediated immune responses to a comparable degree to that of CT-adjuvant. ${ }^{17}$ Importantly, CTA1-DD was found to be completely safe with no side effects even in high doses in mice and nonhuman primates. $^{14}$ While this adjuvant can also function as a delivery platform with an intrinsic adjuvant activity for mucosal vaccines, we generated the CTA1-3M2e-DD fusion protein for further evaluation as a broadly protective universal influenza vaccine. $^{16,18}$ The ectodomain of the influenza M2 protein, the M2e peptide, has been found highly protective in various vaccine formulations, despite that the M2 protein is poorly immunogenic following infection. ${ }^{19}$ Particularly interesting is also that the $\mathrm{M} 2 \mathrm{e}$ peptide is conserved in all human influenza $A$ virus strains and, therefore, it has become the most explored epitope for a universal influenza vaccine and evaluated in several clinical trials. ${ }^{20}$ Previous studies in the mouse model had shown that protection is mediated by anti-M2e antibodies, but recently we could document that also $\mathrm{M} 2 \mathrm{e}$-specific $\mathrm{CD}^{+}{ }^{+} \mathrm{T}$ cells critically contributed to protection against a heterosubtypic influenza virus challenge infection. ${ }^{18,21}$ In fact, we observed a particularly critical role of lung resident M2e-specific memory $\mathrm{CD}^{+}{ }^{+} \mathrm{T}$ cells for protection following i.n. immunization with CTA1-3M2e-DD, which agrees well with recent discoveries of the central role of resident $\mathrm{CD}^{+}{ }^{+} \mathrm{T}$ cells for resistance against infection. ${ }^{18}$

To further improve the immunogenicity of the vaccine vector we investigated whether a combination between LNPs and CTA1$3 \mathrm{M} 2 \mathrm{e}-\mathrm{DD}$ would be beneficial for vaccine efficacy. To this end, the fusion protein was incorporated into the aqueous core, as well as covalently bound to the surface of the LNPs by a thiol-maleimide reaction, with or without adding PEG spacers. We assessed the immunogenicity and protective potential of the combined vector using the influenza mouse model. More specifically, we focused on interactions between the combined vector and DCs and their ability to effectively prime CD4 ${ }^{+}$T-cell immunity and compared the effects with those of the soluble fusion protein given alone in equimolar doses. We found that the nanoparticle formulation strongly enhanced lung protection against a heterosubtypic highly virulent influenza virus challenge infection and that it was the local lung immune response that was superior to that obtained from immunizations with the fusion protein alone. Thus, this is the first proof-of-principle study to demonstrate that an enzymatically active adjuvant can be combined with LNPs to achieve enhanced immunogenicity and dramatically improve protection against infection.

\section{RESULTS}

Combining adjuvant active fusion protein with lipid nanoparticles into novel vaccine vectors

The purpose of the present study was to investigate if combining the highly effective CTA1-DD adjuvant with LNPs could improve the immunoenhancing effect even further compared to that achieved with the fusion protein used alone. To this end we used the CTA1-3M2e-DD (FPM2e) fusion protein, which carries the highly protective $M 2$ e peptide from the ion channel protein $M 2$ of influenza $A$ virus, and evaluated the effect in a mouse model. ${ }^{18}$ To determine the role of the active enzyme, we also constructed LNPs with an enzymatically inactive CTA1R9K-mutant (iFPM2e). ${ }^{13}$ The LNPs were made of POPC, $10 \mathrm{~mol} \%$ of cholesterol, and maleimide-carrying lipids with or without a PEG-2000 spacer to covalently bind the fusion protein by a thiol-maleimide reaction to outwards-facing PEG spacers (FPM2e:LNPPEG) or directly to the non-PEGylated surface (FPM2e:LNP) of the LNPs (Fig. 1a). Three types of constructs were made with the fusion proteins either encapsulated in the core of the LNP or bound to the surface or a combination of the two (Fig. 1a). Nanoparticle tracking analysis (NTA) demonstrated similar size of the PEGylated and nonPEGylated LNPs and a mean hydrodynamic diameter of roughly $150 \mathrm{~nm}$ (Fig. 1b). Both these LNP-types hosted negative zeta potentials at $\mathrm{pH} 7.4$ with $-21.1 \pm 2.61 \mathrm{mV}$ for the PEGylated and $-47.8 \pm 1.62 \mathrm{mV}$ for the non-PEGylated combination (Fig. 1c). For formulations with encapsulated protein, we estimated that $10 \%$ of the fusion protein was entrapped in the core while the rest was surface bound. When we used PEG spacers, we could increase the fraction of surface-bound fusion protein (Fig. 1e). The PEGylated constructs carried $30-40 \%$ more protein than the non-PEGylated constructs, while their sizes were still similar (Fig. 1b, d-f). Stability testing revealed that LNPs were intact for more than 100 days of storage at $4{ }^{\circ} \mathrm{C}$ (Fig. 1g). A Cryo-TEM analysis of the different LNPs showed that the majority of these were unilamellar liposomes and that only $10-20 \%$ were in multilamellar or multivesicular form (Fig. 1h, i).

The combined LNP vector improves antigen presentation by dendritic cells in vitro

First, we evaluated the impact of the combined vaccine vector on the ability of APCs to prime $\mathrm{CD}^{+}{ }^{+} \mathrm{T}$ cells in vitro. We employed A-20 $B$ cells and an M2e-specific T-cell hybridoma that we have developed. ${ }^{22}$ We found that A-20 B cells effectively internalized the fusion protein and presented $\mathrm{M} 2 \mathrm{e}$ peptide to the $\mathrm{T}$ cells (Fig. 2a). LNPs carrying both surface-bound and encapsulated fusion protein were more effective T-cell stimulators, especially in the low dose range where LNP formulations were more immunogenic than the fusion protein alone (Fig. 2a, b). Of note, though, FPM2e alone was already 1000 times more effective compared to an equimolar dose of M2e peptide (Fig. 2b).

Next, we modified the fusion protein so that it carried 3Eapeptides (instead of the $3 \mathrm{M} 2 \mathrm{e}$ peptides), which after complexing with MHC class II (I-A $\left.{ }^{b}\right)$ molecules enabled studies of peptide expression on the surface of the APC. To this end we used an immature splenic DC line, D1, and labeled the peptide-MHC II complex with Y-Ae antibody and analyzed peptide expression by flow cytometry. ${ }^{23}$ We found that the combined CTA1-3Ea-DD (FPEa) LNP vector achieved a higher Ea peptide surfaceexpression level than FPEa alone, as assessed by the mean fluorescence intensity (MFI) of labeled Y-Ae antibody (Fig. 2c). This was particularly evident in the lower dose range at $1 \mathrm{~h}$ of incubation (Fig. 2c, d). A higher expression of MHC class II in the D1 cells coincided with the stronger presentation of Ea-peptide (Fig. 2e). However, between 2 and $4 \mathrm{~h}$ the surface expression of Ea-peptide and MHC class II was gradually reduced and at $4 \mathrm{~h}$ the combined FPEa:LNP vector and FPEa alone were presented equally well for $72 \mathrm{~h}$ or more (Fig. $2 \mathrm{~d}$, e). ${ }^{24}$ Importantly, though, the level of surface expression of the co-stimulatory molecules 
a

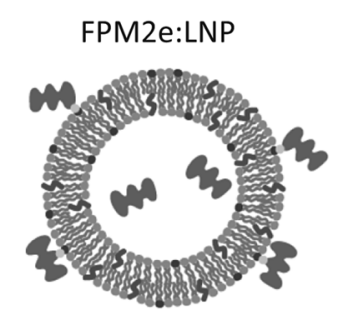

FPM2e:LNPPEG

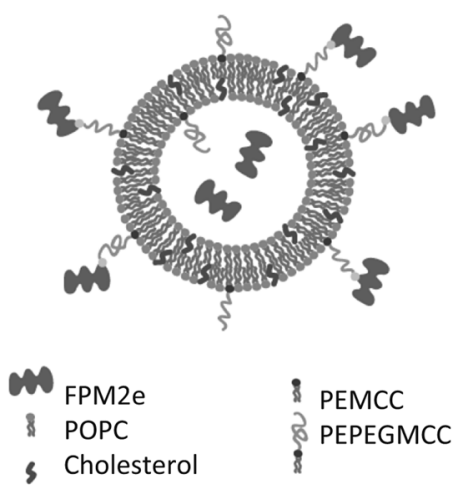

b 2
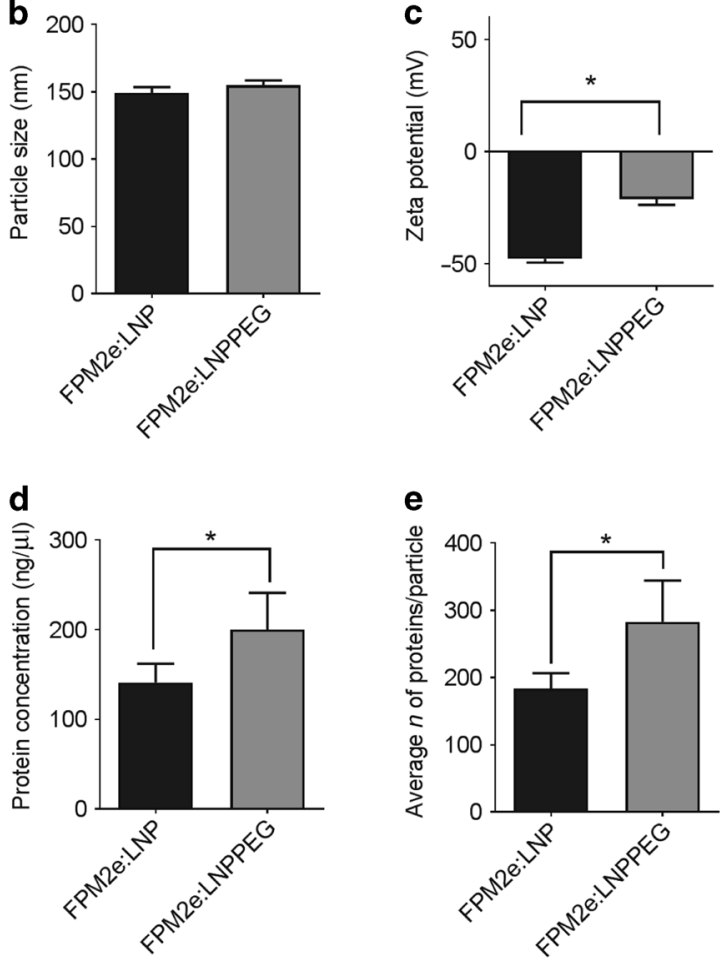

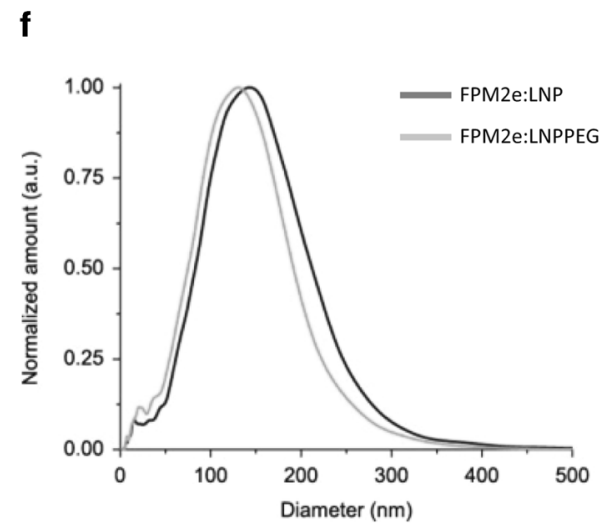

h

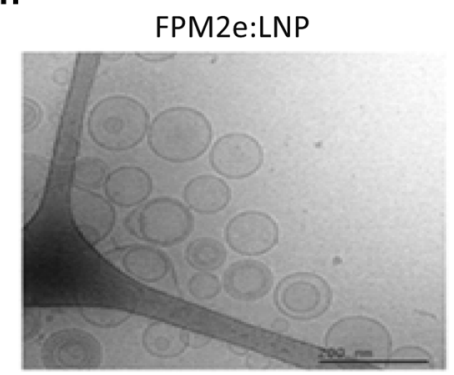

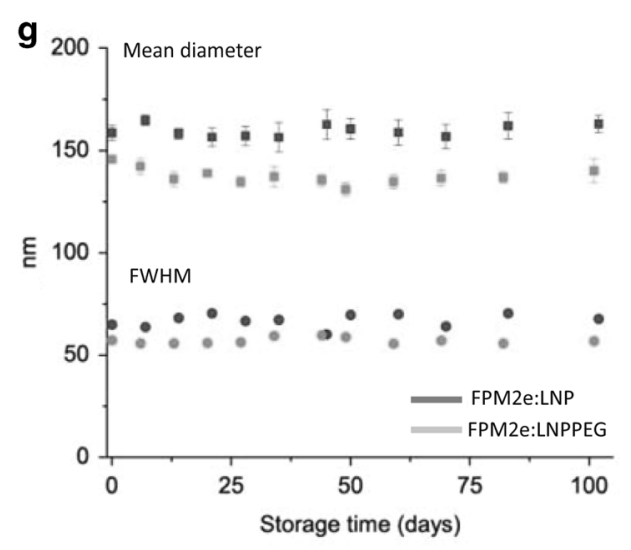

i

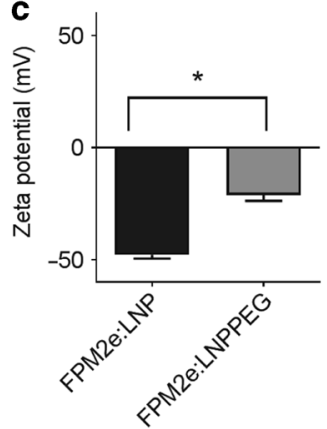

525 
a

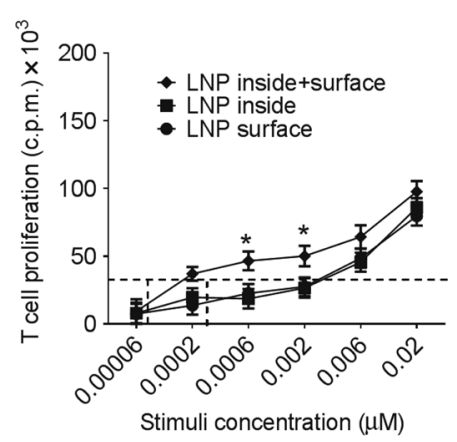

b

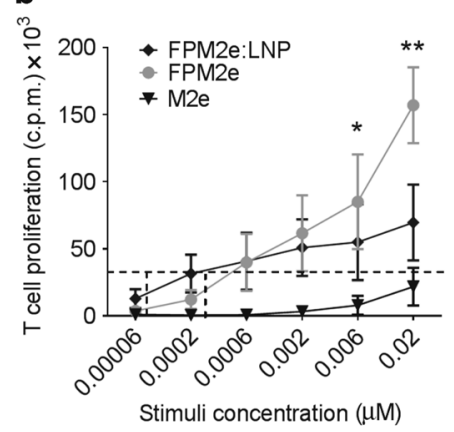

C

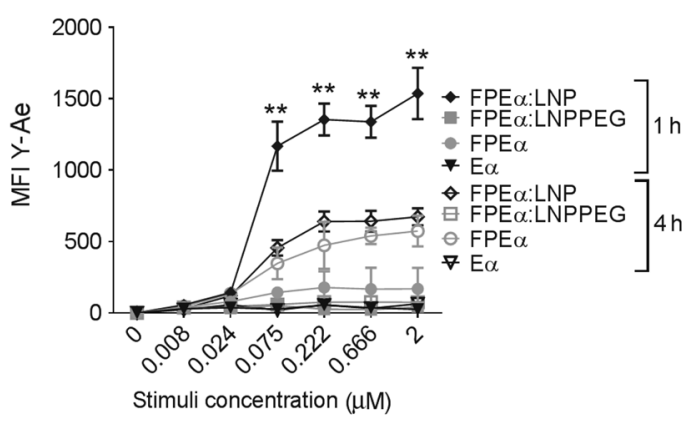

d
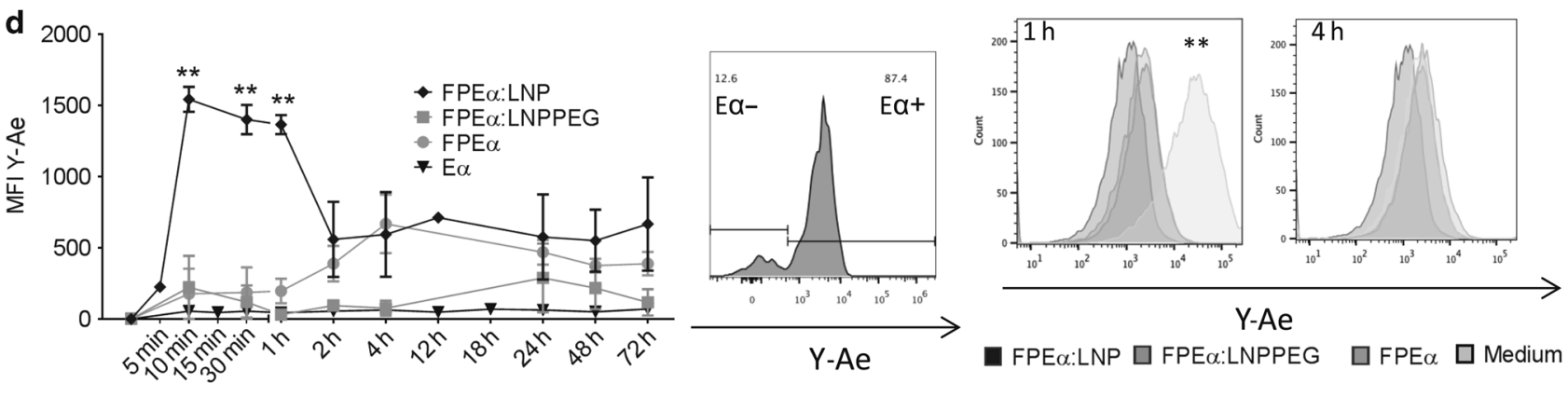

e
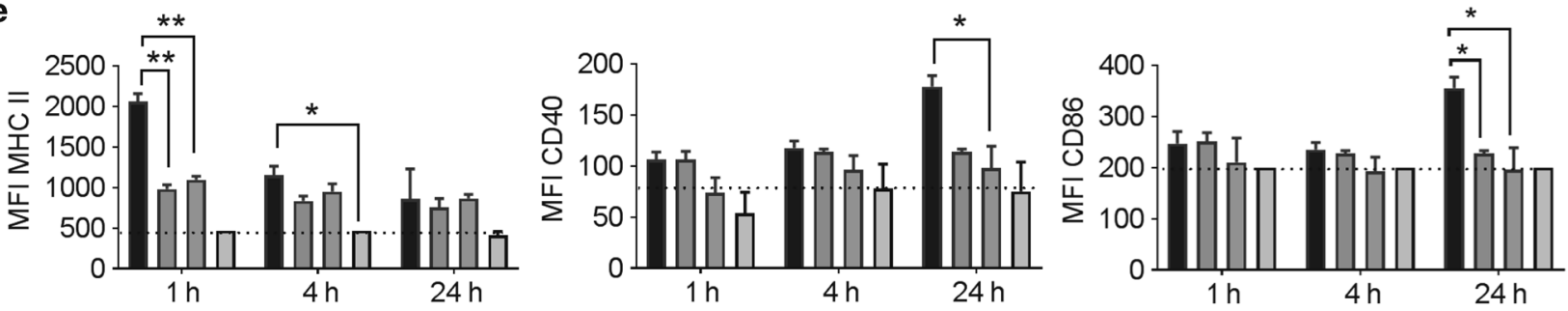

FPE $\alpha: L N P \square$ FPE $\alpha: L N P P E G \quad \square$ FPE $\alpha \square$ Medium

f
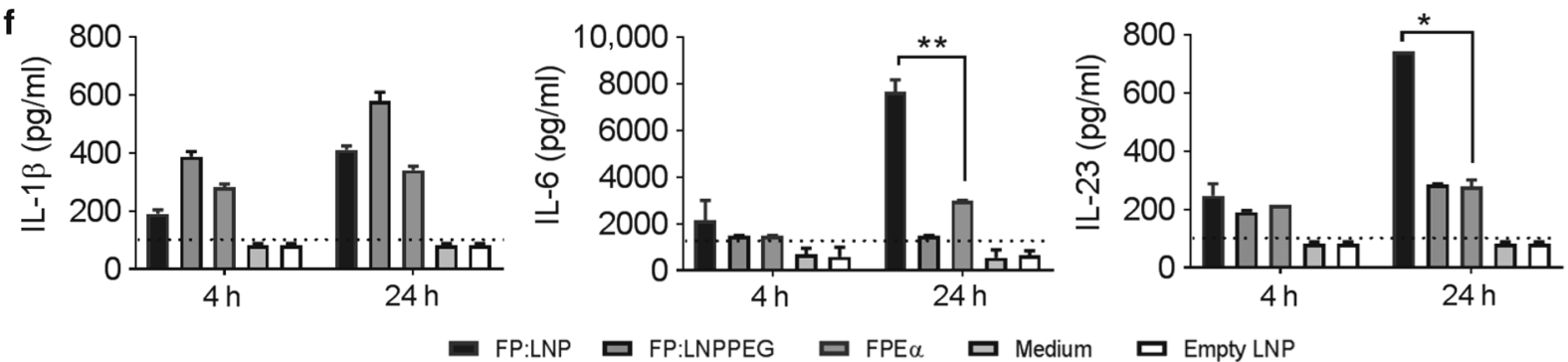

Fig. 2 Uptake and presentation of the combined LNP vector by APCs in vitro. a M2e-hybridoma T-cell activation by A-20 B cells after incubation with a range of concentrations of non-PEGylated LNPs containing CTA1-3M2e-DD in the inner core (LNP inside), having it covalently attached on the surface (LNP surface) or both (LNP inside + surface). IL-2 production was assessed by proliferation of CTLL-2 cells and given as cell proliferation in c.p.m. (counts per minute) \pm SD. The value is the mean of triplicate cultures of each condition in 3 independent experiments. b A similar experimental set up as in (a), but with A-20 B cells incubated with LNPs (inside + outside) of FPM2e, FPM2e:LNP, FPM2e:LNPPEG, or M2e peptide alone. Values are given as mean c.p.m. \pm SD of triplicate cultures of each condition in 3 independent experiments. c The uptake, processing and surface presentation of E $\alpha$ peptide and MHC class II complexes by D1 dendritic cells after incubation for 1 and $4 \mathrm{~h}$ with increasing concentrations of E $\alpha$-peptide, FPE $\alpha$, FPE $\alpha: L N P$, or FPE $\alpha$ :LNPPEG. Surface expression of peptide/ MHC II complexes was determined by geometric mean fluorescent intensity (MFI) of labeled Y-Ae Mab by FACS and plotted as mean \pm SD of triplicate cultures of each condition in 3 independent experiments. $\mathbf{d}$ Same experimental set up as $C$, but with a fixed protein dose of $0,2 \mu \mathrm{M}$ of E $\alpha$-peptide, FPE $\alpha, F P E \alpha: L N P$ or FPE $\alpha: L N P P E G$ analyzed at different time points. Values are given as mean MFI \pm SD of triplicate cultures of each condition in 3 independent experiments (left panel). Representative histograms of Y-Ae MFI for the different conditions after 1 and $4 \mathrm{~h}$ of incubation as indicated (right panels). e Using FACS and labeled antibodies we determined the MFI values of MHC II (left panel), CD40 (middle panel) and CD86 (right panel) expression on D1 cells following incubation with LNPs for $1 \mathrm{~h}, 4 \mathrm{~h}$ and $24 \mathrm{~h}$. Values are given as mean MFI \pm SD of triplicate cultures of each condition in 3 independent experiments. $f$ The production of IL-1 $\beta$ (left panel), IL-6 (middle panel), and IL-23 (right panel) in culture supernatants from (e) was assessed by ELISA and given as $\mathrm{pg} / \mathrm{ml} \pm$ SD. Statistical significance was calculated by unpaired $t$ test and $p$ values are given as ${ }^{*} p<0.05$ and ${ }^{* *} p<0.01$. 
CD40 and CD86 at $24 \mathrm{~h}$ was significantly higher with the combined vector than with FPEa alone (Fig. 2e). Moreover, production of cytokines, IL-6 and IL-23, in particular, was significantly upregulated in D1 cells stimulated with the nonPEGylated combined LNP vector (Fig. 2f). Empty LNP constructs failed to stimulate cytokine production (Fig. 2f). Thus, the nonPEGylated combined LNP vector was found to be the most immunogenic construct in vitro at $24 \mathrm{~h}$ showing the strongest effects on surface peptide expression and co-stimulation with increased membrane CD40 and CD86 levels and production of IL- 6 and IL-23.

Effective targeting and enhanced function of migratory DCs following nasal administration

Following intranasal administration of the combined LNP vector or the fusion protein alone, we observed that migratory $\left(C D 11 c^{+}\right.$, MHC $\left.\|^{\text {high }}\right)$, but not resident $\left(C D 11 c^{+}, M H C \|^{\text {low }}\right)$ DCs in the draining $\mathrm{mLN}$, had taken-up the Ea-peptide and presented it as a membrane complex with MHC class II molecules (Fig. 3a). After $48 \mathrm{~h}$ the LNP formulation was significantly more effective at increasing the total frequency and absolute number of migratory DC in the $\mathrm{mLN}$ (Fig. 3a). Moreover, the LNP immunized mice also exhibited a higher frequency $(10 \%)$ of migratory DC that expressed the Ea-peptide than those immunized with FPa alone (5\%) (Fig. 3a). No Ea-expression was observed in resident DCs indicating that both the combined vector and the soluble FPEa were taken-up in the nasal mucosa by immature DCs rather than being passively transported via lymph or blood to the resident DC population in the $\mathrm{mLN}$ (Fig. 3a). Similar to the in vitro findings we observed upregulated expression of the co-stimulatory molecules CD40, CD80, and CD86 on migratory DCs from LNP-as compared to $\mathrm{FPa}$ alone immunized mice (Fig. $3 \mathrm{a}$ ).

The ability to prime an Ea-specific CD4 ${ }^{+} \mathrm{T}$-cell response in the draining $\mathrm{mLN}$ was evaluated next. After adoptive transfer of CFSElabeled Ea-specific T-cell receptor (TCR) transgenic $\mathrm{CD}^{+}{ }^{+} \mathrm{T}$ cells from B6.Cg-EaTg 3Ayr/J donor mice into C57BL/6 recipient mice, we gave a single i.n. immunization on day 1 with FPEa:LNP or soluble FPEa. Five days later we assessed T-cell priming in the $\mathrm{mLN}$ using flow cytometry and CFSE-dilution in isolated TCR Tg CD4 T cells following immunization (Fig. 3b). We observed strong proliferation of specific CD4 ${ }^{+}$T cells on day 6 in both FPEa:LNP and FPEa-immunized mice (Fig. 3b). The frequency of Ea-specific $\mathrm{CD}^{+}{ }^{+} \mathrm{T}$ cells undergoing cell division was $60-70 \%$ in both immunized groups (Fig. 3b). Interestingly, when TCR Tg CD4 ${ }^{+}$ T cells were injected into the i.n immunized mice at different times this priming ability was maintained for at least 8 days (Fig. 3d). Taken together, the combined FPEa:LNP vector augmented the frequency and absolute number of peptide presenting migratory DCs in the mLN, but, surprisingly, this did not appear to result in a higher priming efficiency of CD4 $\mathrm{T}$ cells in the draining lymph node.

Combined LNP vectors and soluble fusion protein exert comparable systemic immunogenicity

We investigated the impact on M2e-specific antibody production following i.n. immunizations with the combined FPM2e:LNP vector or soluble FPM2e. As M2e-peptide responses are I-A ${ }^{d}$ restricted we used Balb/c mice for these immunizations. ${ }^{21}$ We found that significant M2e-specific lgG antibodies were recorded in serum already after 2 i.n. immunizations with FPM2e:LNP, while FPM2e alone required a third i.n. immunization to achieve a comparable serum IgG antibody response (Fig. 4a). M2e-specific CD4 ${ }^{+}$T-cell $^{-}$ responses were evaluated after 3 i.n. immunizations using recallantigen stimulation in vitro. We observed comparable proliferative responses in the two groups (Fig. 4b). We found that enzymatically inactive mutants iFPM2e:LNP and iFPM2e largely failed to stimulate $\mathrm{CD}^{+}{ }^{-}$T-cell responses (Fig. 4c). Thus, the immunoenhancing effect of the FPM2e:LNP depended on the ADP-ribosylating ability of the CTA1-moiety and was not a consequence of the LNP formulation per se. The M2e-specific cytokine response by $\mathrm{CD}^{+}{ }^{+} \mathrm{T}$ cells to recall-antigen stimulation revealed strong IFN- $\gamma$ production in both FPM2e:LNP and FPM2e-immunized mice, but the soluble protein was more effective at stimulating $\mathrm{CD}^{+}$ T cells producing IL-17 (Fig. 4e, f). Also, PEGylated LNPs, which stimulated lower T-cell proliferation and IFNY-production, surprisingly induced IL-17 production that was similar in magnitude to that seen in mice given FPM2e alone (Fig. $4 \mathrm{~d}-\mathrm{f}$ ). The corresponding serum antibody responses revealed no major differences between the combined FPM2e:LNP vector and FPM2e alone with regard to total anti-M2e lgG, IgG1, or lgG2a antibody levels, whereas PEGylated LNPs gave significantly lower titers (Fig. 4g). Thus, apart from a differential IL-17 response, we could not find a distinct difference at the systemic level between the combined FPM2e:LNP vector and the FPM2e alone. But, these effects required an active CTA1-enzyme and could not be achieved with PEGylated LNPs, albeit IL-17 responses in the latter appeared comparable to those of FPM2e.

Local lung immunity is strongly enhanced by the combined LNP vector

Whereas we found little difference between the combined FPM2e: LNP vector and soluble FPM2e at the systemic level following i.n. immunizations, the local responses were dramatically different. Thus, much stronger $\mathrm{M} 2 \mathrm{e}-\mathrm{specific} \lg \mathrm{A}$ responses in bronchoalveolar lavage (BAL) and threefold increased frequency of $\mathrm{M} 2 \mathrm{e}-$ tetramer-specific $\mathrm{CD}^{+}{ }^{+} \mathrm{T}$ cells in the lung were seen after three i.n immunizations with FPM2e:LNP compared to when FPM2e was given alone (Fig. 5a, b). ${ }^{18} \mathrm{~A}$ dose-response analysis was undertaken to evaluate if repeated doses of the FPM2e:LNP were required to establish the strong $\mathrm{M} 2 \mathrm{e}$-specific $\mathrm{CD} 4^{+} \mathrm{T}$-cell response in the lung. Indeed, we found that whereas a priming immunization hardly resulted in any $\mathrm{M} 2 \mathrm{e}-$-specific $\mathrm{CD} 4^{+} \mathrm{T}$ cells in the lung (1\% of total CD4 T cells) a second (13\%) and third (18\%) dose significantly improved the presence of these cells in the lung (Fig. 5c). The FPM2e:LNP formulation was more effective than the soluble FPM2e alone (Fig. 5c). Moreover, the induced M2e-specific response in the lung hosted both Th1 (IFNY) and Th17 (IL-17) subsets, as determined by ELISPOT analysis of isolated lung CD4 ${ }^{+}$ T cells (Fig. $5 \mathrm{~d}$ ). As before, the PEGylated LNPs failed to stimulate comparable immune responses to those stimulated by nonPEGylated LNPs, but in contrast to systemic responses the local responses were similar to those induced by soluble FPM2e (Fig. 5a-c). M2e-tetramer-specific $\mathrm{CD}^{+} \mathrm{T}$ cells in the lung, as opposed to those from the spleen, were $\mathrm{CXCR}^{+}$and $\mathrm{CD} 69^{+}$, indicating that they were lung resident $\mathrm{CD} 4^{+} \mathrm{T}$ cells, which agrees well with our previous study (Fig. 5e). ${ }^{18}$ Importantly, FPM2e:LNP immunized mice had significantly higher levels and absolute numbers of $\mathrm{CXCR6}^{+}$and $\mathrm{CD}^{+} 9^{+} \mathrm{M} 2 \mathrm{e}$-specific $\mathrm{CD} 4^{+} \mathrm{T}$ cells in the lung compared to FPM2e-immunized mice, suggesting a higher presence of resident memory M2e-specific $\mathrm{CD}^{+} \mathrm{T}^{+}$cells (Fig. 5e). ${ }^{25}$ Taken together, we found that the combined FPM2e: LNP vector was more immunogenic for local respiratory tract $\lg \mathrm{A}$ and $\mathrm{CD}^{+}{ }^{+} \mathrm{T}$-cell responses than the soluble FPM2e fusion protein given alone.

Influenza virus specific lung protection is improved with the combined LNP vector

Because we observed a strong enhancing effect of the local M2especific immune response with the i.n. FPM2e:LNP formulation, we next determined the protective potential against a live influenza virus infection. Following 3 i.n. immunizations with FPM2e:LNP, FPM2e:LNPPEG, or FPM2e alone, we challenged mice i.n. with $4 \times$ $\mathrm{LD}_{50}$ of the reassortant influenza A H3N2 $\times 47$ strain or the H1N1 heterosubtypic PR8 A/Puerto Rico/8/34 strain. ${ }^{26}$ We found that all three immunization regimens gave $80-100 \%$ protection against the $\times 47$ challenge strain (Fig. 6a). By contrast, protection against 
a

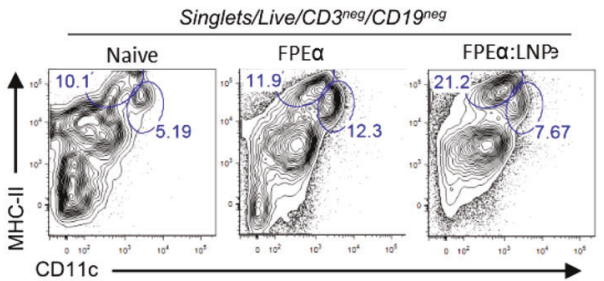

MHC- $-\|^{\text {hi }}$ CD $11 \mathrm{C}^{\text {int }}=$ "migratory DCs" MHC-llint CD11 $\mathrm{C}^{\text {hi }}=$ "resident DCs"
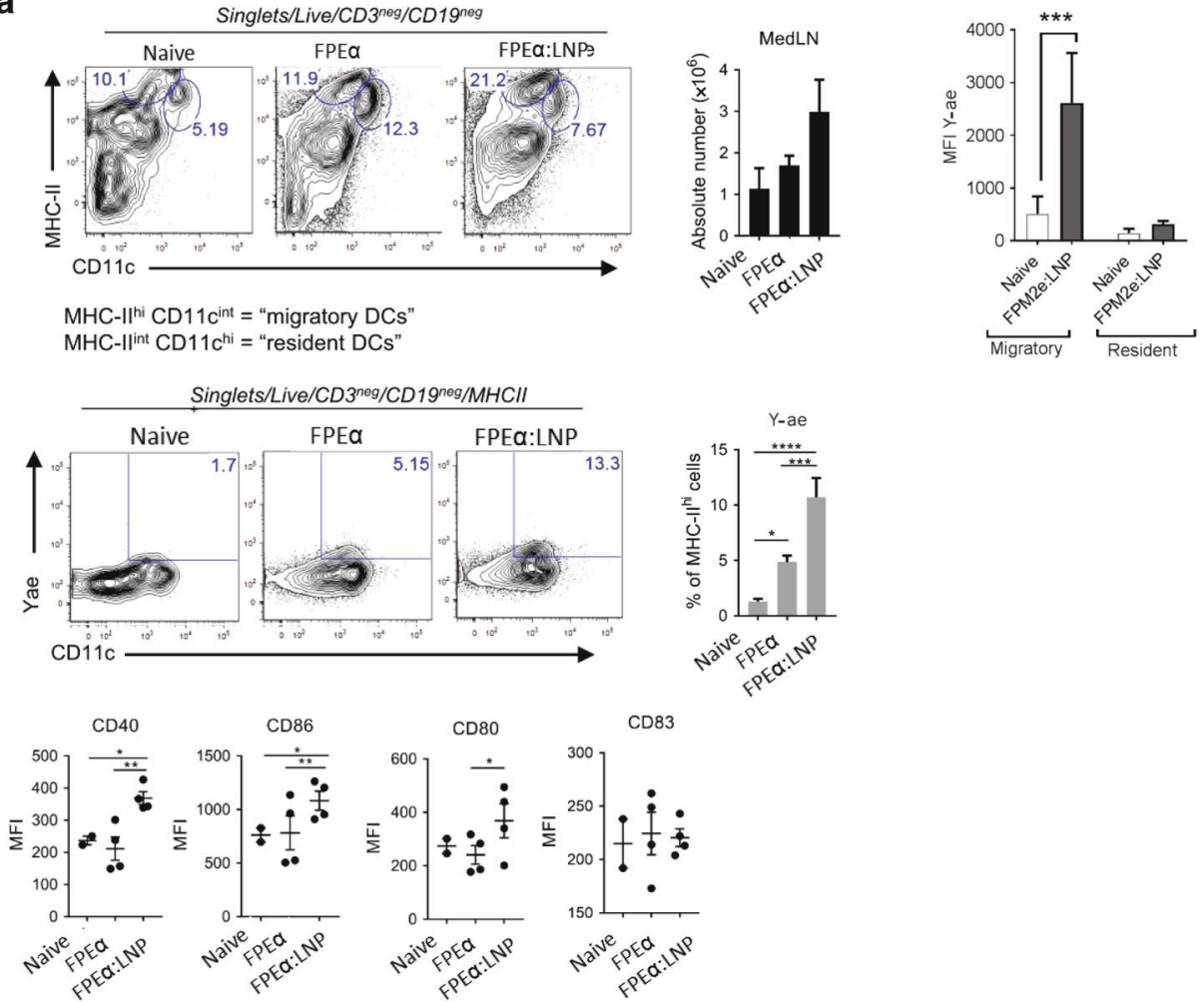

b
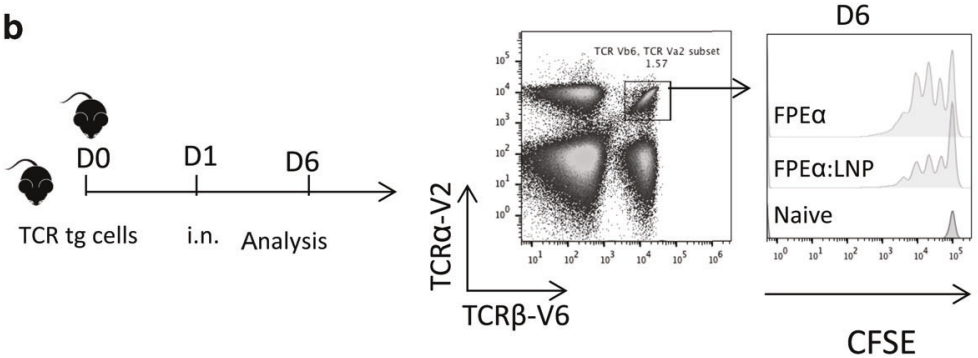

C
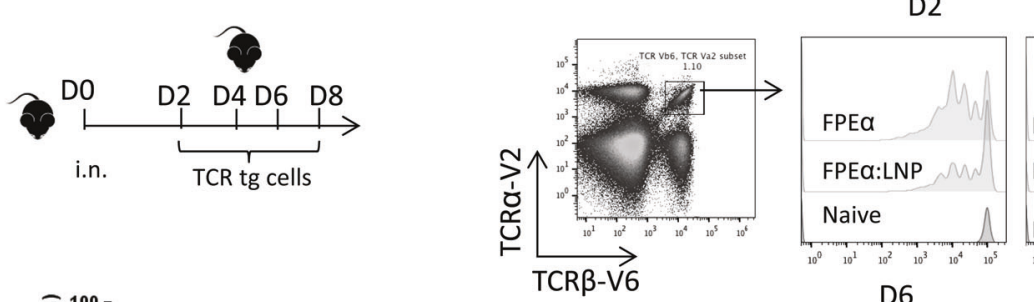

D4

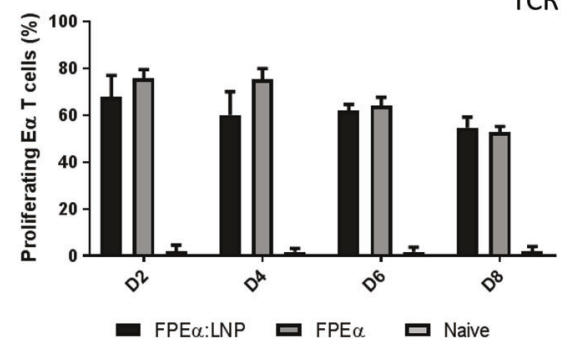

D6

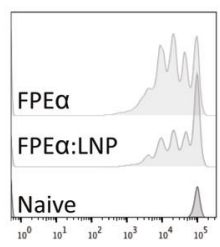

D8

the more aggressive heterosubtypic PR8 strain was achieved only in FPM2e:LNP immunized mice (Fig. 6a, b). Survival was roughly $50 \%$ in this group and less morbidity was observed compared to that found in the FPM2e:LNPPEG or soluble FPM2e-immunized mice (Fig. 6b). Strikingly, lungs from FPM2e:LNP immunized mice exhibited little gross pathology, while unimmunized, FPM2e: LNPPEG or FPM2e-immunized mice showed different degrees of severe lung pathology (Fig. $6 \mathrm{c}$ ). Following the challenge infection FPM2e:LNP immunized mice demonstrated significantly higher levels of lung M2e-tetramer-specific $\mathrm{CD}^{+}{ }^{+}$T cells (Fig. $6 \mathrm{~d}$ ). Because 
Fig. 3 DC uptake and CD4 + T-cell priming ability in vivo following i.n immunizations with the combined LNP vector. a Gating strategy used for identifying migratory and resident DCs in the mediastinal lymph node $(\mathrm{mLN})$ using labeled antibodies against CD11C and MHC II (upper, left panel). Increase in migratory DC after administration of LNPs relative to that seen after use of FPEa alone (upper, middle panel). Uptake of i.n administered FPE $\alpha$ :LNP and FPE $\alpha$ in migratory, but not resident, DCs in the mLN (upper right panel). Representative FACS dot plots of Y-Ae MFI (middle panels) or bars showing the mean expression of co-stimulatory molecules (lower panels) on migratory (MHC II ${ }^{\text {high }}$, CD $11 \mathrm{c}^{+}$) DCs at $48 \mathrm{~h}$ after a single i.n immunization with $10 \mu \mathrm{g}$ FPE $\alpha$ or FPE $\alpha$ :LNP. The mean percentage (\%) of Y-Ae ${ }^{+}$cells or MFI of CD40, CD80 and CD86-expression on migratory DCs was calculated from 3 independent experiments and given as mean \pm SD. b Schematic representation of the experimental protocol used for analyzing CD4 ${ }^{+}$T-cell priming in vivo after adoptive transfer of $2 \times 10^{6}$ CFSE-labeled E $\alpha$ TCR Tg CD4 ${ }^{+} \mathrm{T}$ cells into C57BL/ 6 mice and immunization with $5 \mu \mathrm{g}$ of FPE $\alpha$ or FPE $\alpha$ :LNP (left panel). Gating strategy used to identify proliferating CFSE-labeled $\mathrm{E} \alpha \mathrm{TCR}$ Tg CD4 ${ }^{+} \mathrm{T}$ cells following i.n immunizations (middle panel). Representative FACS histograms of proliferating $\mathrm{E} \alpha \mathrm{TCR}$ Tg CD4 ${ }^{+} \mathrm{T}$ cells in the $\mathrm{mLN}$ on day 6 after a single i.n. immunization (right panel). The percentage of proliferating ( $>2$ cell divisions) CFSE-labeled E $\alpha$ TCR Tg CD4 ${ }^{+}$T cells was calculated and given as means \pm SD of 3 mice in each group and 3 independent experiments (lower panel). c The same model as described in B was used, but the T-cell priming ability in $\mathrm{mLN}$ was assessed at different days after a single i.n. immunization with FPE $\alpha$ or FPEa:LNP (left panel). Gating strategy (middle panel) and representative histograms are given of proliferating CFSE-labeled E $\alpha$ TCR Tg CD4 ${ }^{+}$T cells following injection of cells at 2, 4, 6, and 8 days after a single i.n. immunization with $5 \mu \mathrm{g}$ of FPE $\alpha$ or FPE $\alpha$ : LNP (right panel). The percentage of proliferating ( $>2$ cell divisions) CFSE-labeled E $\alpha$ TCR Tg CD4 ${ }^{+}$T cells was calculated and given as means \pm SD of 3 mice in each group and 3 independent experiments (lower panel). Statistical significance was calculated by unpaired $t$ test and $p$ values are given as ${ }^{*} p<0.05$ and ${ }^{* *} p<0.01$.

we have previously observed a strong protective role of the $\mathrm{M} 2 \mathrm{e}-$ specific CD4 ${ }^{+} \mathrm{T}$ cells, independent of anti-M2e-specific antibodies, we asked whether depletion of $\mathrm{CD} 4^{+} \mathrm{T}$ cells prior to the challenge infection would have a negative effect on protection. ${ }^{18}$ To this end, we depleted $\mathrm{CD}^{+}{ }^{+}$T cells using the GK1.5 Mab in i.n. immunized mice prior to a challenge infection with the PR8 strain. All mice depleted of $\mathrm{CD}^{+}{ }^{+} \mathrm{T}$ cells succumbed to infection, demonstrating that M2e-specific $\mathrm{CD}^{+}{ }^{+} \mathrm{T}$ cells played a critical role for protection (Fig. 6e). Of note, both FPM2e:LNP and FPM2eimmunized mice had similar anti-M2e IgG1 and lgG2a serum-or IgA BAL antibodies, supporting that M2e-specific CD4 T cells strongly contributed to the enhanced protection (Fig. 6f). Thus, the combined FPM2e:LNP vector stimulated stronger local immune responses compared to FPM2e alone with substantially better lung protection, which appeared to be mediated mainly by M2e-specific $\mathrm{CD}^{+}{ }^{+} \mathrm{T}$ cells rather than by serum or BAL anti-M2e antibodies. Hence, the benefit of the combined FPM2e:LNP formulation was much improved antiviral lung protection compared to that found with FPM2e fusion protein given alone. It appeared that an important difference between the two vaccine formulations was the ability to stimulate resident $\mathrm{CXCR6}{ }^{+} \mathrm{CD} 69^{+}$ in M2e-specific CD4 T cells seen with the combined FPM2e:LNP vector.

\section{DISCUSSION}

The present study is a proof-of-principle investigation of whether the efficacy of an already potent self-adjuvanted vaccine, CTA1$3 \mathrm{M} 2 \mathrm{e}-\mathrm{DD}$, can be further improved by combining it with LNPs. We found that this was, indeed, the case. Whereas we found little benefit of combining the fusion protein with LNPs for systemic immune responses, we clearly observed a significantly enhanced local and lung tissue-specific response, involving both $\lg A$ antibodies and lung resident $\left(\mathrm{CD} 9^{+}\right) \mathrm{M} 2 \mathrm{e}$-specific $\mathrm{CD} 4^{+} \mathrm{T}$ cells. These $\mathrm{CD}^{+} \mathrm{T}$ cells critically contributed to protection, as depletion of $\mathrm{CD}^{+}{ }^{+} \mathrm{T}$ cells prior to challenge infection dramatically reduced immune protection and survival in the i.n. immunized mice. Moreover, the combined vector prevented gross lung pathology following a challenge infection as opposed to immunizations with soluble FPM2e and this protective state was associated with a threefold higher frequency of $\mathrm{CCR}^{+} \mathrm{CD}^{+} 9^{+}$ lung M2e-specific $\mathrm{CD}^{+}{ }^{+} \mathrm{T}$ cells, while serum anti-M2e $\operatorname{lgG} 1$ or lgG2a antibodies were comparable to those observed after i.n. immunization with FPM2e alone. The combined vector stimulated higher frequencies of both Th1 and Th17 cells in the lung as compared to soluble FPM2e given i.n. These effects correlated well with the improved functions of FPM2e:LNP-exposed DCs and their priming ability (co-stimulation and cytokine production), leading to a substantially increased level of lung resident M2e-specific $\mathrm{CD}^{+}{ }^{+} \mathrm{T}$ cells. In fact, we observed a $30-50 \%$ increase in the absolute number of resident memory M2e-specific $\mathrm{CD}^{+}{ }^{+} \mathrm{T}$ cells, suggesting that the LNP formulation given i.n specifically promoted lung residency $\left(\mathrm{CXCR6}{ }^{+}, \mathrm{CD}^{+} 9^{+}\right){ }^{25,27}$ Furthermore, the stronger lung Th1- and Th17 responses were likely the effect of an upregulated CD40 and CD86 co-stimulation and enhanced cytokine production (IL-6 and IL-23) by the targeted DCs. This effect required the active ADP-ribosylating CTA1-enzyme because the inactive mutant, CTA1(R9K)-3M2e-DD, failed to affect the migratory DCs. ${ }^{18}$ This is consistent with our earlier studies, showing that the adjuvant effect resided in the CTA1 enzymatic function and that the LNP formulation itself did not act as an adjuvant, but rather provided a vehicle for antigen delivery. ${ }^{28}$ Thus, the LNP formulation did not appear to compensate for the reduced immunogenicity of the inactive mutant iFPM2e. However, recently we changed the lipid composition of the LNP into a 1,2distearoyl-sn-glycero-3-phosphocholine (DSPC)-based gel phase liposome, rather than a fluid phase liposome, and combined the fusion protein with these LNPs. ${ }^{29}$ This change revealed some inherent immunomodulating properties of the LNP formulation itself in vitro, but more importantly it potentiated the CTA1adjuvant effects even further and improved antigen presentation and co-stimulation by the DCs. Whether the change of lipid composition of the LNPs also can enhance immunogenicity and protective immunity in vivo awaits to be tested.

Different strategies to help nanoparticles penetrate the nasal mucosal barrier have been presented earlier. ${ }^{3}$ For example, the inclusion of PEG, chitosan (deacetylated chitin), alginate, polyvinyl alcohol, hyaluronan, or cellulose derivatives in the nanoparticle have all been reported effective in preventing aggregation and facilitating diffusion across the mucosal barrier. We decided to incorporate PEG because it has previously been suggested to reduce the particle-retention time at mucosal surfaces. ${ }^{30,31}$ However, PEG was originally used to increase retention time in the circulation and avoid adsorption of plasma proteins to the LNP. ${ }^{31}$ Our study did not find a beneficial effect of including PEGylation as a means to increase immunogenicity, although it enabled an increased binding of FPM2e fusion protein to the LNP. Rather the opposite was observed and the fusion protein appeared to lose immunogenicity because an equimolar dose of soluble FPM2e alone gave better antigen presentation in targeted DCs in vitro, as well as higher specific serum lgG antibody levels compared to FPM2e:LNPPEG. Thus, our findings are in conflict with several previous publications showing positive effects of PEGylation on the immunogenicity of LNPs. ${ }^{32}$ For example, a derivative of PEG was successfully used to avoid entrapment in mucus and to increase membrane penetration in an intravaginal 
a

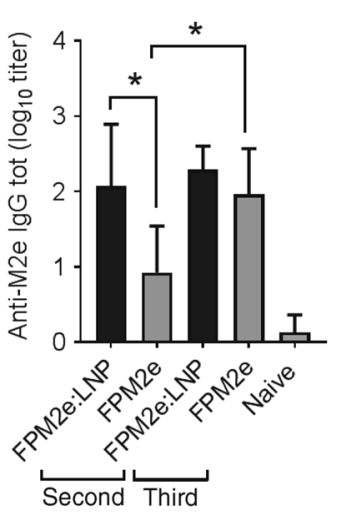

d

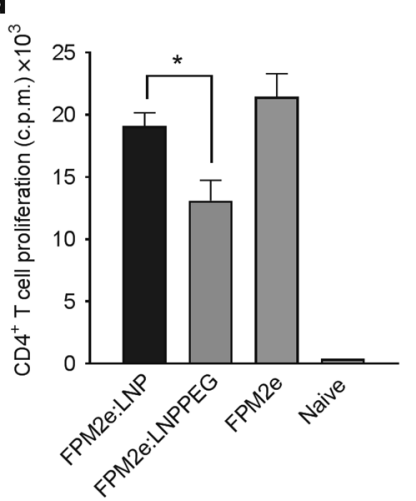

$\mathbf{f}$

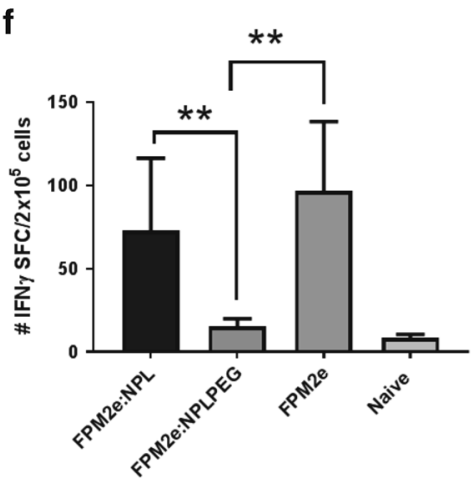

g

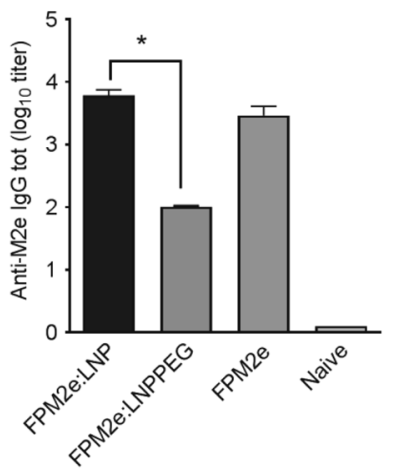

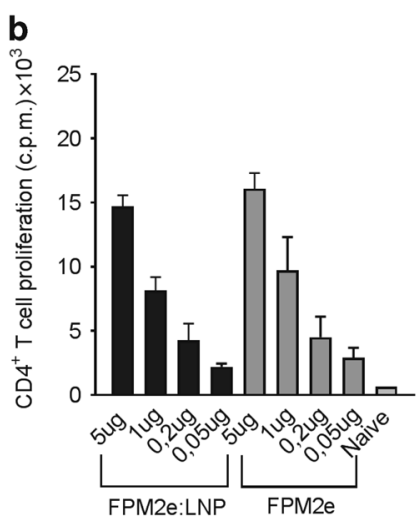
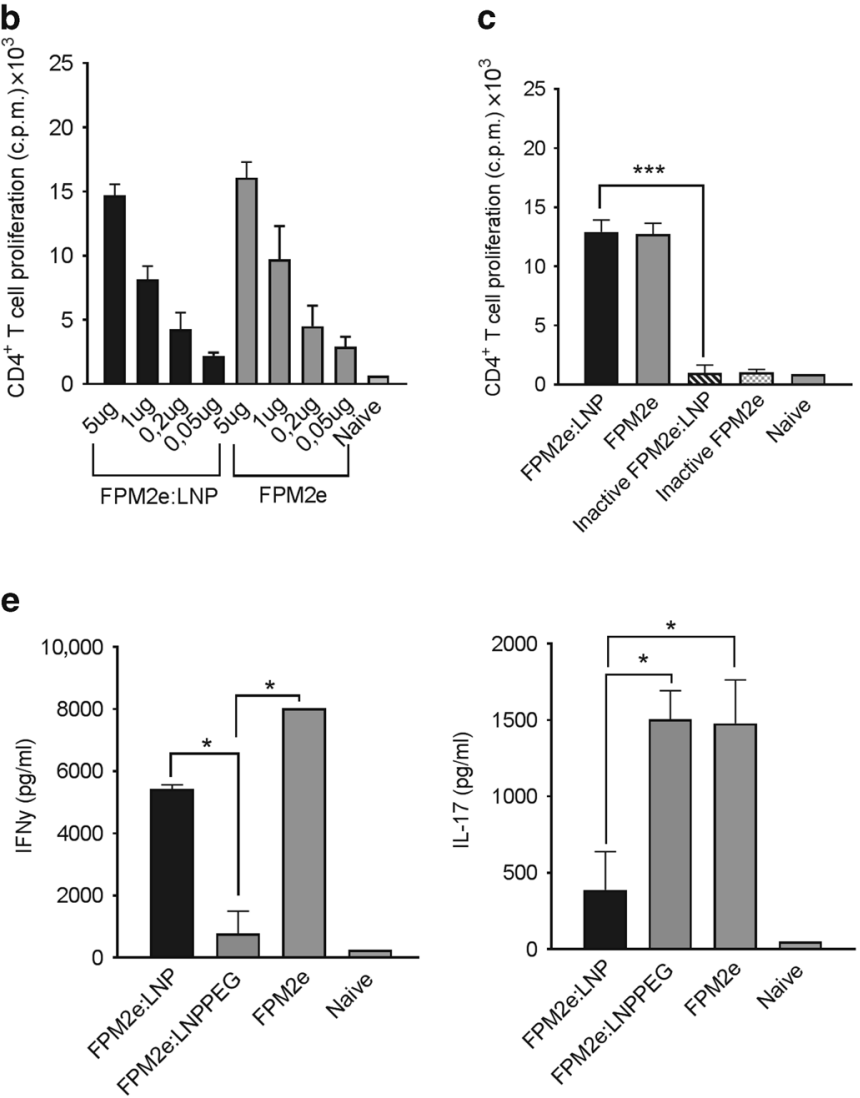

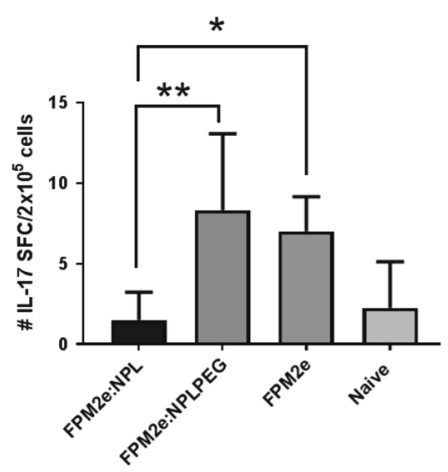

vaccine formulation. ${ }^{33}$ Whereas PEGylation is used to reduce interactions with mucus, cells and soluble proteins and in this regard it could be expected that DCs in vitro do not take up antigen as effectively as with non-PEGylated LNPs, albeit studies in vivo have suggested the opposite, namely that there is a positive correlation between the degree of PEGylation and an increased immune priming effect. ${ }^{34}$ So, it is still difficult to predict if and how PEGylation can benefit a mucosal LNP-based vaccine. 
Fig. 4 No difference in systemic immunogenicity between the LNP formulation and that of soluble FPM2e. a Balb/c mice were immunized i.n at 10 days apart with 2 or 3 doses with $5 \mu \mathrm{g}$ FPM2e or FPM2e:LNP and serum M2e-specific IgG antibody titers were assessed by ELISA at 10 days after the last dose. $\log _{10}$ titers are given as means \pm SD of 5 mice in each group and 3 independent experiments giving similar results. b-f Recall responses to M2e peptide in cultured splenocytes following 3 i.n immunizations with a dose range of FPM2e or FPM2e:LNP, as indicated in B. Or assessed after 3 i.n immunizations with $5 \mu \mathrm{g}$ of FPM2e, FPM2e:LNP, inactive FPM2e or inactive FPM2e:LNP, as indicated (c-f). ${ }^{3} \mathrm{H}$-Thymidine uptake was assessed and values are given for proliferating CD4 ${ }^{+} \mathrm{T}$ cells in triplicates as mean cpm $\pm \mathrm{SEM}$ of groups of 5 mice. This is one representative experiment out of 3 independent experiments giving similar results (b-d). The production of IFN- $\gamma$ (left panel) or IL$17 \mathrm{~A}$ (right panel) in culture supernatants was assessed by ELISA and given as $\mathrm{pg} / \mathrm{ml} \pm \mathrm{SD}$ (e). Individual IFN- $\gamma$ (left panel) or IL-17A (right panel) producing CD4 ${ }^{+} \mathrm{T}$ cells in culture were assessed by ELISPOT and given as mean SFC $/ 2 \times 10^{5}$ cells \pm SD. g Serum M2e-specific total IgG (left panel), lgG1 (middle panel), or lgG2a (right panel) antibodies were determined by ELISA in groups as indicated and given as mean log titers \pm SD of one representative experiment out of 3 giving similar results. Statistical significance was calculated by unpaired $t$ test and $p$ values are given as ${ }^{*} p<0.05,{ }^{* *} p<0.01$, and ${ }^{* * *} p<0.005$.

Nevertheless, we do not know if a sufficient density of PEGylation of our LNPs was achieved for improved immunogenicity. ${ }^{35}$ Hence, further studies are needed to address this question.

There are many ways of incorporating antigens into LNPs. Antigens can be hosted in the aqueous core of the LNP, inserted into the membrane leaflet or bound to the surface by covalent bonds or intermolecular forces. Thus, the LNP formulation may be tailored for the specific needs and purposes. ${ }^{2,36}$ We made LNPs with the fusion protein bound to the surface, as well as incorporated inside the LNP. This was also shown in earlier studies to be better, as cholera toxin B-subunit (CTB) was found to be more effective when bound to the surface than encapsulated inside the LNP. In fact, Rincon-Restrepo et al. showed that antigens encapsulated within the core of a liposome were better primers of $\mathrm{CD}^{+} \mathrm{T}$ cells and antibody responses, while LNP surface exposed antigens preferentially stimulated cytotoxic $\mathrm{CD}^{+} \mathrm{T}$ cells. ${ }^{37}$ Targeting the early endosomal compartment $\left(\mathrm{EEA} 1^{+}\right)$was found effective for $\mathrm{CD}^{+}{ }^{+}$T-cell priming, as we also observed in the present study (unpublished Bernasconi et al.), while presence of antigen in the lysosomal $\left(\mathrm{LAMP}^{+}\right)$compartment rather favored $\mathrm{CD}^{+}{ }^{+}$cells. $^{37}$ Accordingly, we could speculate that the immunomodulating effect of the CTA1-enzyme, at least partially, occurs in the early endosome of the DC.

We found an enhanced ability of DCs to process and present antigen delivered by the combined LNP vector and a higher density of membrane peptide:MHC class II complexes was observed both in vitro and in vivo. This increased performance also included higher expression of co-stimulatory molecules and enhanced cytokine production, which likely contributed to the significantly better stimulation of lung homing M2e-specific $\mathrm{CD}^{+}$ $\mathrm{T}$ cells following i.n immunizations. Indeed, we observed a threefold stronger lung $\mathrm{CD}_{4}^{+} \mathrm{T}$-cell response with the combined FPM2e:LNP vector than with the FPM2e alone. By contrast, the systemic responses detected in the $\mathrm{mLN}$ were comparable between the two. We do not know the exact mechanism responsible for the enhanced local lung $\mathrm{CD}^{+}{ }^{+}$T-cell promoting effect, but the stronger CD86 co-stimulation could have contributed to an enhanced lung homing effect via CXCR6 ${ }^{+}$. Indeed, CXCR6-expression has been found to correlate with lung tissue localization of $\mathrm{CD}^{+} \mathrm{T}$ cells and it is likely to have a similar effect on $\mathrm{CD}^{+}{ }^{+}$T-cell localization. ${ }^{25,27}$ Also, the $30-50 \%$ higher level of CD69-expressing M2e-specific $\mathrm{CD}^{+}{ }^{+} \mathrm{T}$ cells in the lung argues in favor of that the LNP formulation promoted lung residency in primed $\mathrm{CD}^{+}{ }^{+} \mathrm{T}$ cells. Alternatively, repeated i.n immunizations with FP/LNPs may have promoted peptide presentation by lung DCs or alveolar macrophages (AM) that locally could have promoted tissue residency, but our preliminary investigations into such a possibility failed and we could not detect lung DCs or AM with significant peptide presentation (unpublished observation Bernasconi et al). Nevertheless, a majority of the migratory DCs from the nasal cavity to the $\mathrm{mLN}$ are classical CD11 $\mathrm{c}^{+} \mathrm{DCs}$, that can be separated into the three subsets, $C D 103^{+} C D 11 b^{-}$, also termed $\mathrm{CDC} 1$ cells, and $\mathrm{CD} 103^{+} \mathrm{CD} 11 \mathrm{~b}^{+}$or $\mathrm{CD} 103^{-} \mathrm{CD} 11 \mathrm{~b}^{+} \mathrm{DCs}$, termed CDC2 cells. ${ }^{38}$ Whereas, CTA1-DD can bind all these subsets we speculate that $\mathrm{CDC1}$ cells are the most critical target population, but $\mathrm{CD} 103^{+} \mathrm{CD} 11 \mathrm{~b}^{+} \mathrm{CDC} 2$ cells could also be involved in the $\mathrm{CD}^{+}$T-cell priming. ${ }^{39}$ In fact, a previous study demonstrated that CD103-antibody-targeting of conjugated antigen to mucosal DCs by i.n. administration showed strong priming of $\mathrm{CD}^{+}{ }^{\mathrm{T}}$-cell responses, albeit CDC1 cells are best known for crosspriming $\mathrm{CD}^{+} \mathrm{T}$ cells, as seen, for example, in response to influenza infection. ${ }^{40,41}$ Because resident DCs did not exhibit any Ea-peptide following i.n. immunization, we concluded that migratory DCs are the actively priming DC population in the $\mathrm{mLN}$ following i.n. immunizations. ${ }^{42}$

The M2e ectodomain is highly conserved across all human influenza A viruses. ${ }^{43,44}$ Protection generated by $\mathrm{M} 2 \mathrm{e}-$ immunizations largely relies on anti-M2e $\lg G$ and, more specifically, IgG2a antibody production in the BALB/c mouse model. ${ }^{45}$ However, we could recently show that M2e-specific memory $\mathrm{CD}^{+} \mathrm{T}$ cells were induced following i.n. immunization and these contributed to protection, as assessed in Balb/B congenic mice. ${ }^{18}$ We found that mice which failed to develop M2e-specific $\mathrm{CD}^{+}{ }^{+} \mathrm{T}$-cell immunity succumbed to infection, despite having adequate and comparable serum anti-M2e IgG2a titers for protection. ${ }^{18}$ Also when we tested B-cell-deficient mice, we found some protection in i.n. immunized mice after a challenge infection. ${ }^{18}$ In the present study we observed stronger stimulation of M2e-tetramer-specific lung $\mathrm{CD}^{+}{ }^{+} \mathrm{T}$ cells with FPM2e:LNP and elimination of these $\mathrm{CD}^{+}{ }^{+} \mathrm{T}$ cells prior to the challenge infection was detrimental to survival, supporting a critical role for these cells in protection. The FPM2e:LNPs-enhanced lung IFN-y and IL-17 response resulted in resistance against a highly virulent PR8 strain. It was clear from the gross pathology of the lungs of surviving mice that seemingly no tissue destruction had occurred in the lungs of FPM2e:LNP immunized mice. Less well protected mice that succumbed to the infection in the FPM2e, FPM2e:LNPPEG, or unimmunized groups exhibited very severe tissue destruction and lung hemorrhage.

More broadly protective influenza vaccines are much needed. Preclinical studies have indicated that i.n. vaccines are superior to injectable vaccines for the stimulation of local $\lg A$ antibody production and tissue-specific resident $\mathrm{CD}^{+}$and $\mathrm{CD}^{+}{ }^{+}$T-cell immunity. ${ }^{1}$ It is, therefore, encouraging that this proof-of-concept study has documented a strong local protective effect of the combined FPM2e:LNP vector. We believe the flexibility of the combined vector system is particularly interesting because it also allows novel protective vaccine antigens to be included in the LNP and/or inserted as peptides in the fusion protein. We have previously combined CTA1-3M2e-DD with immune stimulating complexes (ISCOMs) to achieve a particulate vaccine formulation, with improved immune stimulating properties when given i.n. ${ }^{21}$ However, while ISCOMs are stable, protein-containing cage-like structures composed of cholesterol, phospholipids and saponins from the Quillaja saponaria Molina tree, they have limitations in their antigen loading capacity and may also be toxic due to the immunomodulating saponins. ${ }^{46}$ The LNP technology explored in the present study provides a much more versatile and dynamic particle and it can be anticipated that including a selected set of 
a

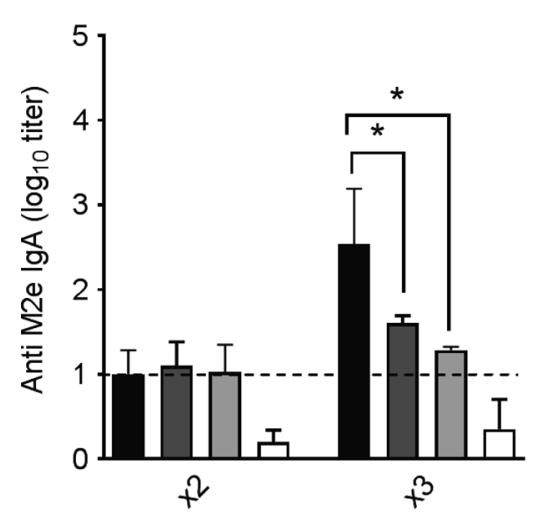

C

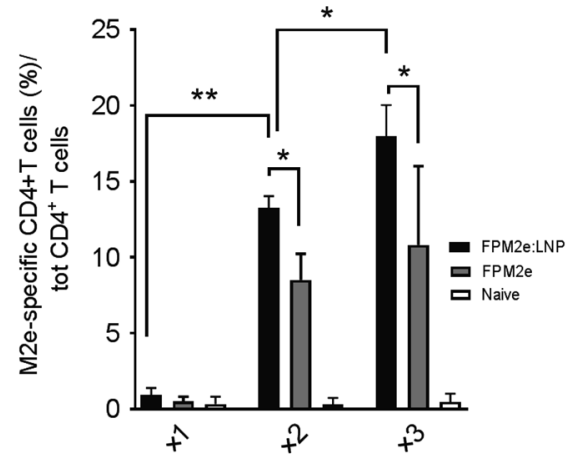

e

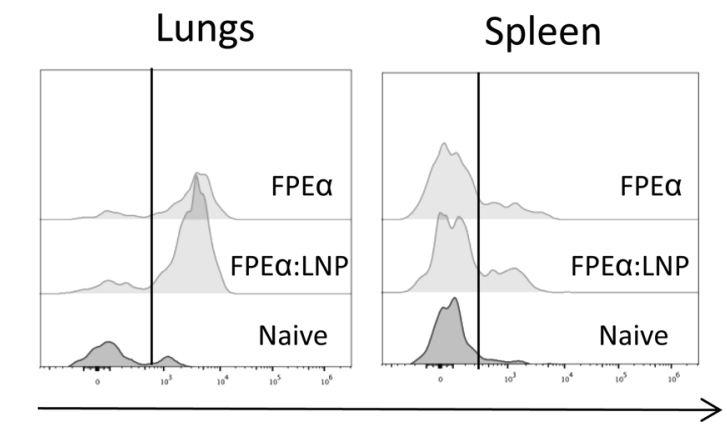

CXCR6
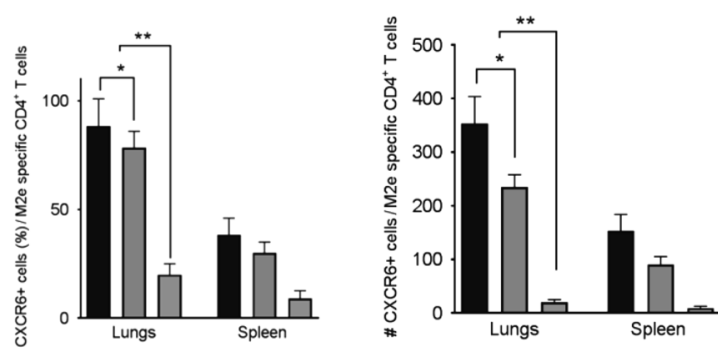

CXCR6

d b
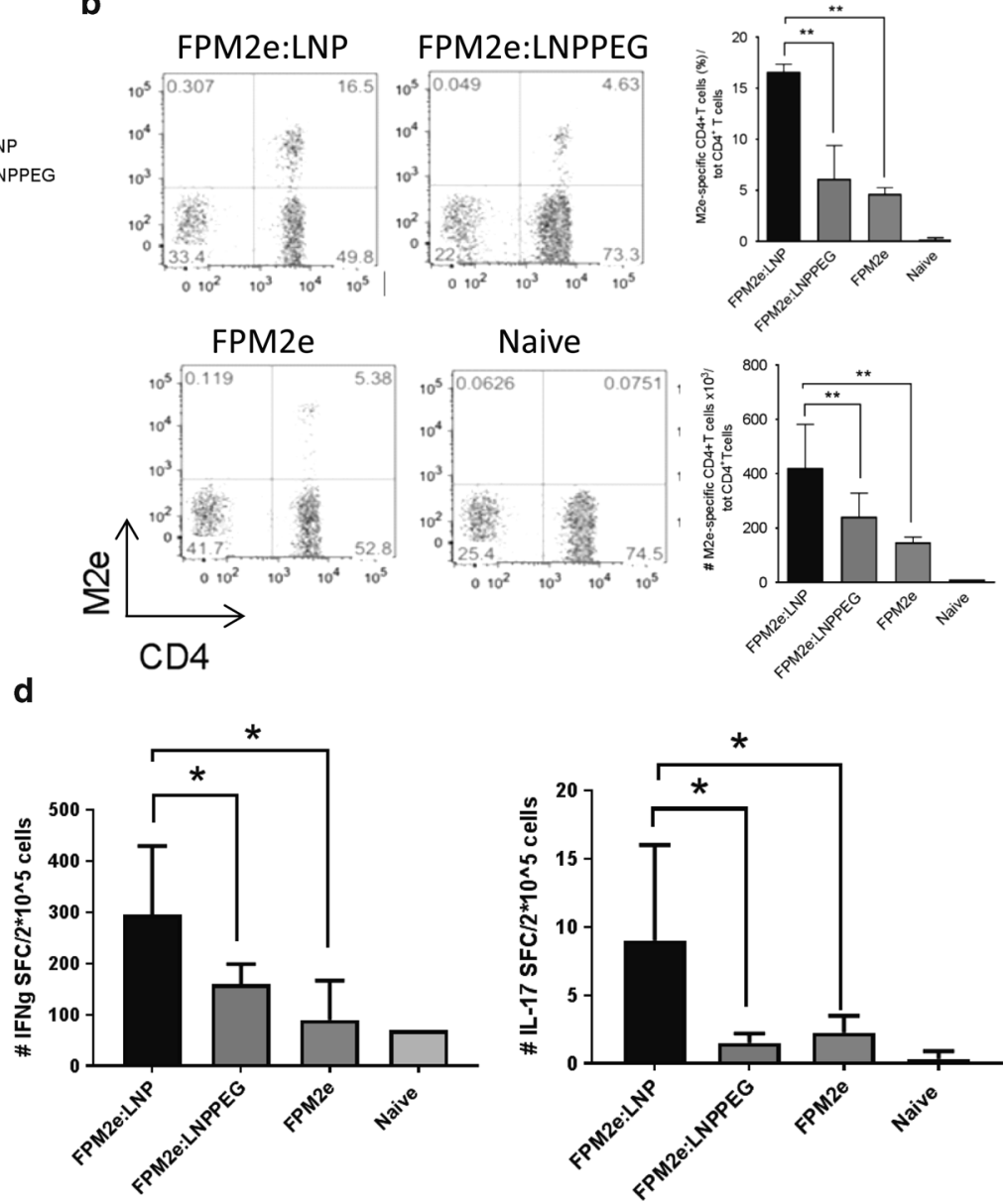

Lungs

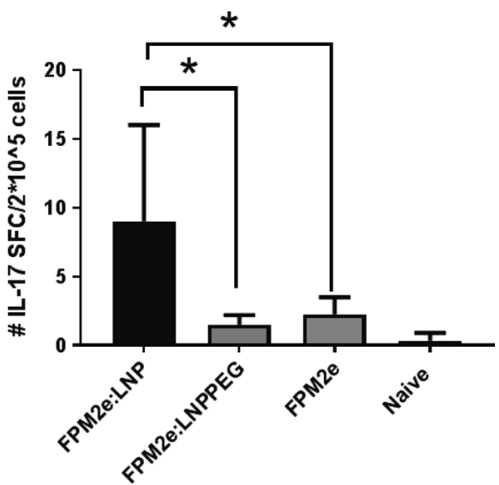

Spleen

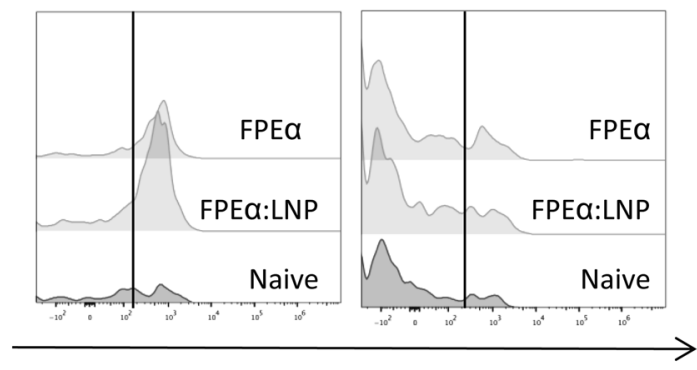

CD69

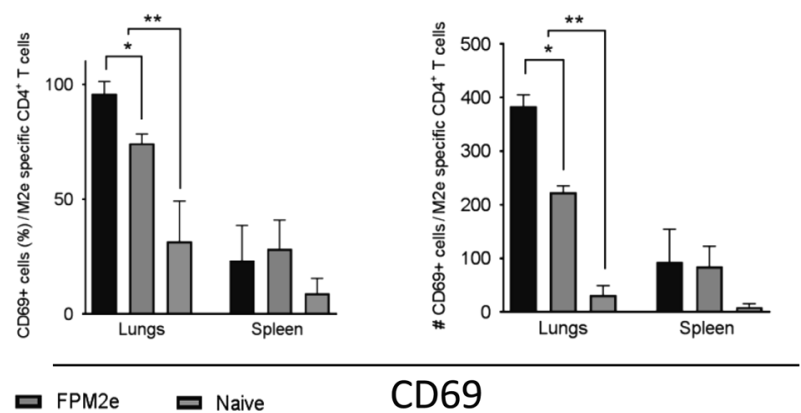

influenza vaccine. The present study also conveys optimism as to the protective ability of this novel combined vector as a generic platform for mucosal vaccines against many other infectious diseases. relevant epitopes and changing the lipid composition of the LNP could further improve the protective efficacy against newly emerging or genetically drifted influenza virus strains. Hence, this could be a way forward toward a broadly protective universal 
Fig. 5 Significant enhancement of local respiratory tract immunogenicity of FPM2e formulated in LNPs. a Balb/c mice were i.n immunized with 2 and 3 doses, respectively, given at 10 days apart with $5 \mu \mathrm{g}$ FPM2e, FPM2e:LNP or FPM2e:LNPPEG and M2e-specific IgA antibodies in BAL were assessed by ELISA 10 days after the final immunization. No significant increase in specific $\operatorname{lgA}$ in BAL after 2 doses were recorded. The values after 3 immunizations are given as mean $\log _{10}$ titers \pm SD of 5 mice in each group and one representative experiment of 3 giving similar results. b Detection of M2e-tetramer-specific CD4 ${ }^{+}$T cells in the lung of immunized mice. Representative FACS dot plots of M2e-tetramer ${ }^{+}$ $\mathrm{CD}^{+} \mathrm{T}$ cells in the different groups from one representative experiment out of 3 giving similar results (left panel). The percentage (right upper panel) and absolute number (right lower panel) of antigen primed M2e-tetramer ${ }^{+} \mathrm{CD}^{+} \mathrm{T}$ cells are given as means \pm SD of 5 mice and 3 independent experiments. c A kinetic dose-response analysis of the M2e-tetramer-specific CD4 ${ }^{+} \mathrm{T}$ cells in the lung following a single, two or three doses of FPM2e:LNP or FPM2e and means \pm SD values are given in $\%$ of at least two independent experiments. d Individual lung CD4 T cells producing IFN- $\gamma$ (left panel) or IL-17A (right panel) to recall M2e-stimulation was determined by ELISPOT and values are given as mean SFC $/ 2 \times 10^{5}$ cells \pm SD of 5 mice and one representative experiment out of 3 giving similar results. e Detection of M2e-tetramer-specific CD4 ${ }^{+}$ T cells expressing CXCR6 and CD69 in the lungs and spleen of i.n immunized mice. Representative FACS histograms of M2e-tetramer ${ }^{+}$CD4 $^{+}$ T cells in the different groups from one representative experiment out of 3 giving similar results (upper panels). The percentage (lower panel) and absolute number (lower panel) of antigen primed M2e-tetramer ${ }^{+} \mathrm{CD}^{+}{ }^{+}$cells expressing CXCR6 or CD69 are given as means \pm SD of 5 mice and 3 independent experiments. Statistical significance was calculated by unpaired $t$ test and $p$ values are given as ${ }^{*} p<0.05$ and ${ }^{* *} p<0.01$.

\section{MATERIAL AND METHODS}

Mice and immunizations

Age- and sex-matched BALB/C or C57BL/6 mice were obtained from Harlan (The Netherlands) or Janvier Laboratories (France), while the Ea-specific TCR transgenic B6.Cg-Tg(TCRa,TCR $\beta) 3 A y r / J$ mice were obtained from The Jackson Laboratories (USA). All mice were maintained at the Laboratory for Experimental Biomedicine (University of Gothenburg, Sweden) under specific pathogen-free conditions. Experiments were ethically approved by the University of Gothenburg ethical committee. Immunization protocol is describe in. ${ }^{16}$ Mice were sacrificed after the final immunization or virus challenge infection and spleens, $\mathrm{mLN}$, serum and $\mathrm{BAL}$ were collected. Serum and BAL were taken stored at $-20^{\circ} \mathrm{C}$ until further analysis.

\section{Fusion protein construction}

CTA1(C189A)-3M2e-DD, with enzymatic activity, CTA1(R9K)-3M2e$\mathrm{DD}$, the enzymatically inactive mutant, CTA1-DD and CTA1 (C189A)-3Ea-DD were produced in Escherichia coli by MIVAC Development $A B$, Sweden, as previously described in refs. ${ }^{21,16}$ The enzymatic ADP-ribosyltransferase activity was determined by the NAD:agmatine assay, ${ }^{47}$ protein analysis and concentrations were determined as described in ref. ${ }^{16}$

\section{Nanoparticle preparation}

Lipid vesicles composed of 1-palmitoyl-2-oleoyl-sn-glycero-3phosphocholine (POPC), cholesterol and1,2-distearoyl-sn-glycero3-phosphoethanolamine- $\mathrm{N}$-[maleimide(polyethylene glycol)-2000] (PEPEGMCC) (89:10:1 mol\%) or POPC, cholesterol and 1,2-dioleoylsn-glycero-3-phosphoethanolamine- $\mathrm{N}$-[4-(p-maleimidomethyl) cyclohexane-carboxamide] (PEMCC) (85:10:5 mol\%) were prepared by the lipid film hydration and extrusion method. Briefly, lipids diluted in a methanol/chloroform (1:1 v/v) mixture were added to a round-bottom flask. The solvents were evaporated first under reduced pressure ( $200 \mathrm{mbar})$ in a $50^{\circ} \mathrm{C}$ water bath for $30 \mathrm{~min}$ and then under vacuum overnight. The thin lipid films were rehydrated in $\mathrm{NaAc}$ saline $(10 \mathrm{mM}$ sodium acetate, $150 \mathrm{mM} \mathrm{NaCl}$, $\mathrm{pH}=5.0$ ) containing $0.16 \mathrm{mg} / \mathrm{mL}$ of fusion protein to a final total lipid concentration of $8 \mathrm{mM}$. The solutions were then further diluted with $\mathrm{NaAc}$ saline to a lipid concentration of $4 \mathrm{mM}$ and extruded 11 times through two $100 \mathrm{~nm}$ nucleopore track-etched polycarbonate membranes (Whatman, UK) using a mini extruder (Avanti Polar Lipids Inc, USA). Unincorporated fusion protein was removed using Amicon Ultra (Millipore, USA) $100 \mathrm{kDa}$ cutoff centrifugal filters as follows: $200 \mu \mathrm{L} \mathrm{NaAc}$ saline and $200 \mu \mathrm{L}$ lipid vesicle solution was added to each filter and centrifuged $(5 \mathrm{~min}$, $8000 \mathrm{rcf}, 10^{\circ} \mathrm{C}$ ) followed by a further dilution with $200 \mu \mathrm{L} \mathrm{NaAc}$ saline in each filter and another centrifugation ( $5 \mathrm{~min}, 8000 \mathrm{rcf}$, $\left.10^{\circ} \mathrm{C}\right)$. Filters were inverted and centrifuged $\left(1 \mathrm{~min}, 8000 \mathrm{rcf}, 10^{\circ} \mathrm{C}\right)$ to recover the solution.
The fusion protein was covalently bound to the liposomes using a thiol-maleimide reaction. Briefly, Traut's reagent $0.02 \mathrm{mg} / \mathrm{mL}$ in PBS $(137 \mathrm{mM} \mathrm{NaCl}, 2.7 \mathrm{mM} \mathrm{KCl}$ and $10 \mathrm{mM}$ phosphate, $\mathrm{pH} 7.4$ (Sigma Aldrich, Sweden)) with $2 \mathrm{mM}$ EDTA, $\mathrm{pH}=7.8$ ) and fusion protein $(1.6 \mathrm{mg} / \mathrm{mL}$ in $10 \mathrm{mM} \mathrm{NaH} 2 \mathrm{PO} 4,0.16 \mathrm{M} \mathrm{NaCl}$, pH 7.4) were mixed to a volume ratio of 5:3 and allowed to react for $20 \mathrm{~min}$ at $4{ }^{\circ} \mathrm{C}$. The vesicles recovered after filtering $200 \mu \mathrm{L}$ were added to $250 \mu \mathrm{L}$ of freshly thiolated fusion protein and incubated for $1 \mathrm{~h}$ at room temperature with gentle shaking. Unreacted fusion protein was removed using Amicon Ultra (Millipore, USA) $100 \mathrm{kDa}$ cutoff centrifugal filters as follows: $125 \mu \mathrm{L} \mathrm{NaAc}$ saline and approximately $290 \mu \mathrm{L}$ LNP solution was added to each filter and centrifuged ( $5 \mathrm{~min}, 8000 \mathrm{rcf}, 10^{\circ} \mathrm{C}$ ) followed by a further dilution with $150 \mu \mathrm{L}$ $\mathrm{NaAc}$ saline in each filter and another centrifugation (5 min, 8000 $\mathrm{rcf}, 10^{\circ} \mathrm{C}$ ). Filters were inverted and centrifuged ( $1 \mathrm{~min}, 8000 \mathrm{rcf}$, $10^{\circ} \mathrm{C}$ ) to recover the solution. LNP solutions were stored at $4{ }^{\circ} \mathrm{C}$.

Size, zeta potential, and protein quantification

The size distributions of the LNPs were determined using NTA, which was performed with a NanoSight LM10 (NanoSight Ltd, UK) equipped with a $630 \mathrm{~nm}$ laser and a Marlin F-033B camera (Allied Vision Technologies, Germany). Each sample was measured at least 5 times (measurement duration: $60 \mathrm{~s}$, camera shutter: 1480, camera gain: 680) after exchanging solution in between. Analysis of the captured movies was performed with NTA software version 2.3 using the following settings: screen gain: 10, detection threshold: 5, blur: $7 \times 7$, minimum track length: automatic, minimum expected particle size: $30 \mathrm{~nm}$. NTA measurements were performed over a period of more than 100 days in order to assess the stability of the LNPs.

Liposome concentration was determined using a NanoSight LM10 (Malvern, UK) equipped with a Hamamatsu C11440-50B/ A11893-02 camera and a $488 \mathrm{~nm}$ laser. A series of three different dilutions was measured five times each, with the solution exchanged in between each 60 -s measurement, and the mean particle concentration was calculated. Analysis was performed using NTA software version 3.2 with camera level: 12 and detection threshold: 2 .

The zeta potentials of the LNPs (in 2 mM HEPES buffer, $\mathrm{pH}=7.4$ ) were measured at $25^{\circ} \mathrm{C}$ in a DTS1070 folded capillary cell using a Zetasizer Nano ZS (Malvern, UK). The viscosity and refractive index of the dispersant were set to $0.8872 \mathrm{CP}$ and 1.33 , respectively. The LNP refractive index was set to 1.45 .

The fusion protein content was determined using the CBQCA Protein Quantitation Kit (Thermo Fisher Scientific, Sweden) as per manufacturer's instructions, using a $5 \mathrm{mM}$ stock solution of CBQCA reagent and addition of $0.1 \%$ Triton X-100 (Sigma Aldrich, Sweden) to the reaction buffer. The measurements were done using a FLUOstar OPTIMA microplate reader (BMG Labtech, Germany) (excitation: $440 \mathrm{~nm}$, emission: $520 \mathrm{~nm}$ ). 

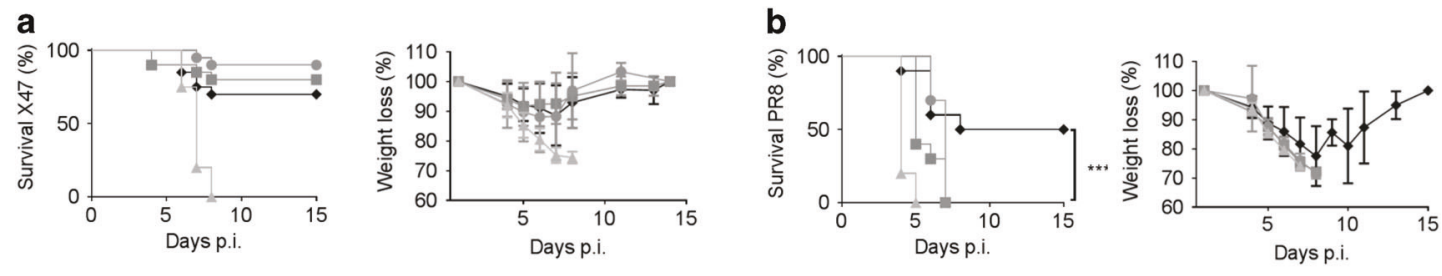

$\rightarrow$ FPM2e:LNP $=$ FPM2e:LNPPEG - FPM2e $=$ Naive

- FPM2e:LNP = FPM2e:LNPPEG * FPM2e $\star$ Naive

C

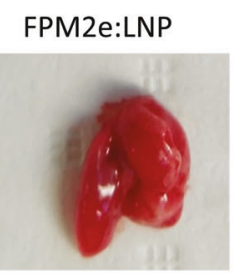

FPM2e:LNPPEG

FPM2e

Naive
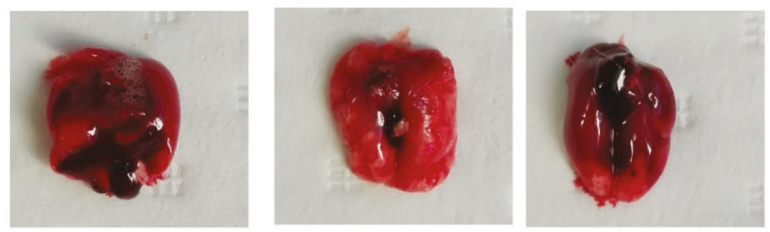

d

FPM2e:LNP

FPM2e:LNPPEG
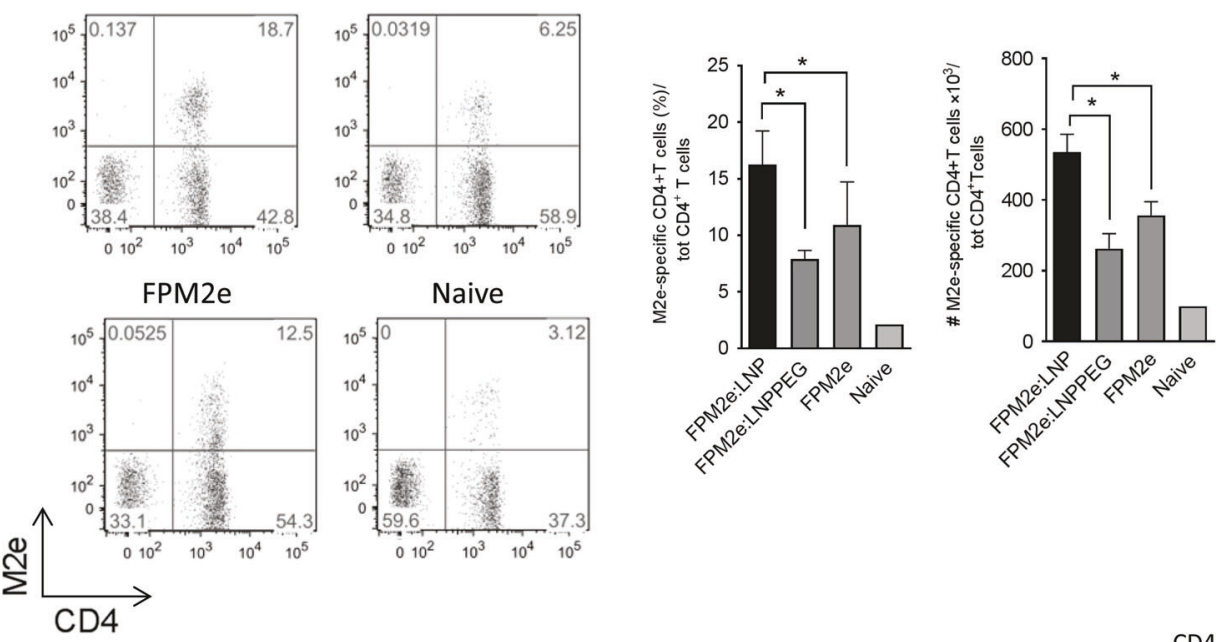

e
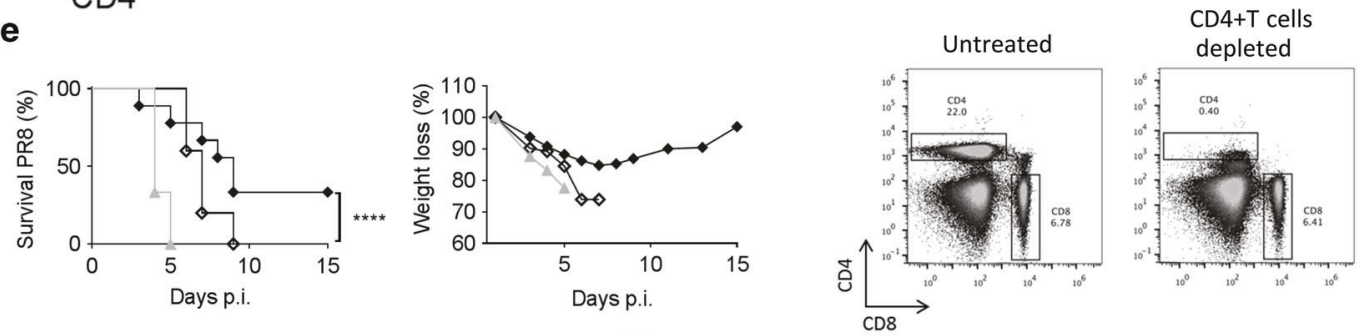

- FPM2e:LNP $\diamond$ FPM2e:LNP CD4+T cells depleted $\approx$ Naive
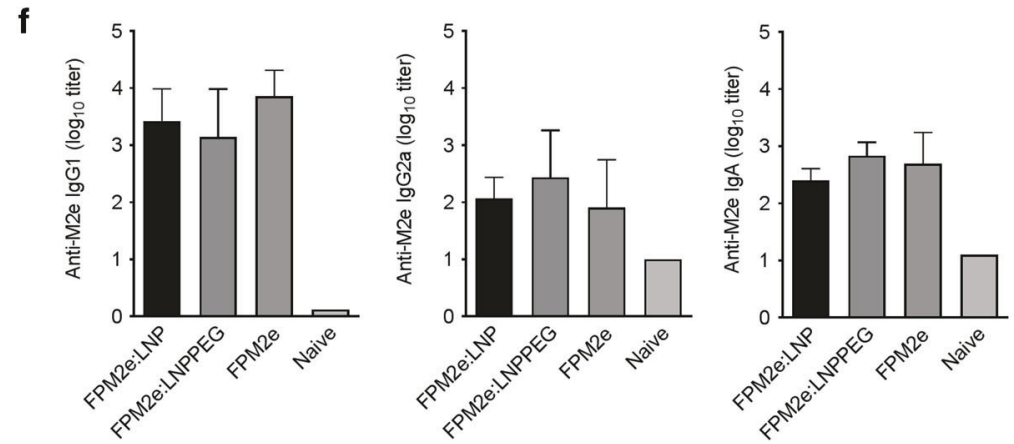

In order to assess the amount of material lost during vesicle production, lipid vesicles of the above-mentioned compositions but also containing $0.5 \mathrm{~mol} \%$ of Lissamine Rhodamine B 1,2-Dihexadecanoyl-sn-Glycero-3-Phosphoethanolamine (Rhod-PE)

were produced. The lipid ratios were assumed to be constant throughout the production process. Absorbance of Rhod-PE was measured using a QM-4/2005 spectrofluorometer (Photon Technology International Inc., USA) (all slits: $4 \mathrm{~nm}$ ). The number of vesicles in 
Fig. 6 Enhanced lung protection following immunization with the combined FPM2e:LNP vector. Balb/c mice were i.n immunized with 3 doses with $5 \mu \mathrm{g}$ FPM2e, FPM2e:LNP, or FPM2e:LNPPEG at 10 days apart and survival and weight loss was monitored following a challenge infection with $4 \times$ LD50 of the mouse adapted $\times 47$ (a) or PR8 (b) influenza A virus strains. The percent of surviving mice (left panels) and body weight loss (right panels) are given of groups of 10 mice and one representative experiment of 3 giving similar results is shown. c Representative images of the gross pathology of the lung in the different groups of mice at 7 days post infection with the PR8 strain. d Detection of M2e-tetramer-specific $\mathrm{CD}^{+}{ }^{+} \mathrm{T}$ cells in the lung of immunized and PR8-challenged mice. Representative FACS dot plots of M2etetramer ${ }^{+} \mathrm{CD}^{+} \mathrm{T}$ cells in the different groups from one representative experiment out of 3 giving similar results (left panel). The percentage

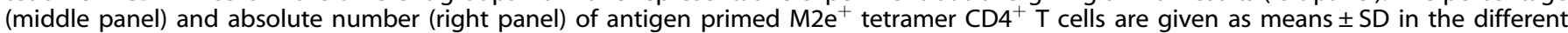
groups of 5 mice and one representative experiment is shown out of 3 giving similar results. e Percent of surviving mice (left panel) and body weight loss (middle panel) following depletion of $\mathrm{CD}^{+}{ }^{+} \mathrm{T}$ cells and challenge infection with $4 \times$ LD50 of PR8 virus strain. FACS plot showing successful depletion of $\mathrm{CD}^{+}$T cells in lungs (right panel). $\mathbf{f}$ Anti-M2e antibodies assessed in the different treatment groups: serum titers of IgG1(left panel) or lgG2a (middle panel) or IgA titers (right panel) in BAL were determined by ELISA after the PR8 infection and given as mean $\log _{10}$ titers \pm SD of 3 independent experiments. Statistical significance was calculated by unpaired $t$ test and $p$ values are given as ${ }^{*} p<0.05$; ${ }^{* *} p<0.01 ;{ }^{* *} p<0.005 ;{ }^{* * * *} p<0.0001$.

the final solution was estimated using geometrical considerations as follows: a liposome was assumed to be a spherical double lipid layer of the average diameter determined by NTA containing lipids with a footprint of $0.68 \mathrm{~nm}^{2}$. The molecular weight of the fusion protein is estimated to be $45 \mathrm{kDa}$.

Cryo-transmission electron microscopy

In order to achieve a suitable concentration for cryogenic transmission electron microscopy (Cryo-TEM), LNP solutions were concentrated in Amicon Ultra $100 \mathrm{kDa}$ cutoff centrifugal filters $\left(8000 \mathrm{rcf}, 10^{\circ} \mathrm{C}\right)$ until one third of the volume remained. Samples for electron microscopy were prepared in a controlled environment vitrification system to ensure stable temperature and to avoid loss of solution during sample preparation. The samples were prepared as thin liquid films, $<300 \mathrm{~nm}$ thick, by applying $5 \mu \mathrm{L}$ of LNP solution to lacey carbon filmed copper grids and blotting away the excess liquid. The samples were immediately plunged into liquid ethane at $-180^{\circ} \mathrm{C}$. This leads to vitrified specimens, avoiding component segmentation and rearrangement, and water crystallization, thereby preserving original microstructures. The vitrified samples were stored under liquid nitrogen until measured. An Oxford CT3500 cryoholder (Oxford Instruments PLC, UK) and its workstation were used to transfer the sample into the Philips CM120 BioTWIN Cryo electron microscope (Philips, Netherlands) equipped with a post-column energy filter (Gatan GIF100; Gatan, Inc., USA). The acceleration voltage was $120 \mathrm{kV}$. The images were recorded digitally with a CCD camera under low electron dose conditions.

In vitro antigen presentation assays

The A-20 murine lymphoma B-cell line, IL-2-dependent murine cell T line CTLL-2 and D1 cell line were used. The M2e-specific T-cell hybridoma was obtained following fusion of in vitro stimulated lymphocytes. ${ }^{18}$ A-20 cells, M2e-hybridoma cells and D1 cells were cultured as described in ref. ${ }^{16}$, either alone or with limiting dilution starting from $0.2 \mu \mathrm{M}$ M2e peptide (Pepscan, Netherlands), CTA1-3M2e-DD or CTA1-3M2e-DD formulated into nanoparticles. IL-2 biologic activity was determined by the IL-2-dependent proliferation of the CTLL-2 cells. CTLL-2 cells were cultured for $24 \mathrm{~h}$ in the presence of supernatant. During the last $5 \mathrm{~h}$, the cultures were pulsed with [3H]-thymidine (PerkinElmer, USA). The [3H]thymidine uptake was determined using a scintillation-beta counter (Beckman, LKB, Sweden).

To assess the processing efficiency of fusion protein we determined the cell surface expression of peptide plus MHC II complex as described in ref. ${ }^{16}$

Antigen processing by migratory DCs and CD4+ T-cell priming in vivo

Four to six weeks old, age and sex-matched TCR transgenic B6.Cg$\mathrm{Tg}(\mathrm{TCRa}, \mathrm{TCR} \beta) 3 \mathrm{Ayr} / \mathrm{J}$ mice were immunized i.n. with $50 \mu \mathrm{g}$ of protein using the fusion proteins alone or incorporated into LNPs.
At $24 \mathrm{~h}$ after a single i.n. administration of fusion protein or LNPs, mice were sacrificed and the $\mathrm{mLN}$ were extracted and single cell suspensions were prepared. The level of Ea-loaded MHC II molecules on isolated migratory DCs was assessed as described in ref. ${ }^{16}$ Alternatively, C57BL/6 mice were immunized intranasally (i.n.) $8,6,4$, and 2 days before the cell transfer and $\mathrm{mLN}$ were analyzed 3 days post transfer. Half of the mice were randomly assigned to receive either (1) an i.n. administered dose of Fingolimod (FTY720, Sigma Aldrich, Sweden) $(100 \mu \mathrm{g} / \mathrm{mouse})$ in $200 \mu \mathrm{L}$ PBS, or (2) an equally large volume of PBS every 48 ho (days $1,3,5$, and 7). Proliferating $\mathrm{CD}^{+}{ }^{+} \mathrm{TCR} \mathrm{Va2}{ }^{+}{\mathrm{V} \beta 6^{+}}^{+}$cells were identified by reduced CFSE-staining.

Influenza virus challenge experiment

Influenza virus challenge experiments were performed using a lethal i.n. dose of $4 \times$ LD50, of PR8 A/Puerto Rico/8/34 (H1N1) virus or the mouse adapted $\times 47$ virus (a reassortant between $A$ / Victoria/3/75 (H3N2) and A/Puerto Rico/8/34 (H1N1)). Morbidity (body weight) and mortality were monitored daily for 2 weeks as described in ref. ${ }^{16}$

\section{$\mathrm{CD}^{+}{ }^{-}$-cell immune responses}

We assessed the $\mathrm{CD} 4^{+}$T-cell response after immunizations by flow cytometry using the PE-labeled M2e-tetramer, designed for the study by the NIH Tetramer Core Facility (Bethesda, USA) as described in ref. ${ }^{16}$ Moreover, we used M2e peptide in vitro to recall responses in single cell suspensions from spleen and $\mathrm{mLN}$ from immunized and control mice as described in ref. ${ }^{16}$

Antibody responses

Serum and BAL were collected from individual mice and M2e- and $\mathrm{HA}$-specific $\lg$ and $\lg \mathrm{A}$ antibody were measured by ELISA as described in ref. ${ }^{16}$

Statistical analysis

Statistical analysis was performed in Prism (GraphPad Software) using unpaired $t$ test. All reported $p$ values are two sided and values of $<0.05$ were considered to indicate statistical significance. ${ }^{*} p<0.05 ;{ }^{* *} p<0.01 ;{ }^{* *} p<0.005 ;{ }^{* * * *} p<0.0001$.

\section{ACKNOWLEDGEMENTS}

The study received funding from the People Programme (Marie Curie Actions) of the European Union's Seventh Framework Programme FP7/2007-2013 under REA grant agreement number 607690 . It was also supported in parts by research funds from the Knut and Alice Wallenberg Foundation KAW 2013.0030, the Swedish Foundation for Strategic Research SB12-0088, The Swedish Cancer Foundation, The Swedish Research Council, the EU project UNISEC, LUA/ALF ALFGBG-531021 and the Lundberg foundation. The authors would like to thank Jan-Olof Andersson and Richard Christison at MIVAC Ab for of the fusion proteins production. Special thanks to the NIH Tetramer Core Facility for producing the unique MHC class II restricted M2e tetramer. We also thank staff at the Laboratory for Experimental Biomedicine at the University of Gothenburg. Moreover, we thank Gunnel Karlsson at Biomicroscopy 
Unit, Polymer and Materials Chemistry, Chemical Centre, Lund University, Lund, Sweden for the Cryo-TEM work.

\section{AUTHOR CONTRIBUTIONS}

V.B., K.N., G.L., M.B., F.H., and N.L. designed the study and planned the experiments. V.B., K.N., S.B., N.P., K.S., and A.S. performed the experiments and analyzed the data. V.B., K.N., and N.L. made the figures. V.B., K.N., and N.L. wrote the paper.

\section{ADDITIONAL INFORMATION}

Competing interests: The authors declare no competing interests.

Publisher's note Springer Nature remains neutral with regard to jurisdictional claims in published maps and institutional affiliations.

\section{REFERENCES}

1. Lycke, N. Recent progress in mucosal vaccine development: potential and limitations. Nat. Rev. Immunol. 12, 592-605 (2012).

2. Bernasconi, V., Norling, K., Bally, M., Hook, F. \& Lycke, N. Y. Mucosal vaccine development based on liposome technology. J. Immunol. Res. 2016, 5482087 (2016).

3. Corthesy, B. \& Bioley, G. Lipid-based particles: versatile delivery systems for mucosal vaccination against infection. Front. Immunol. 9, 431 (2018).

4. Pati, R., Shevtsov, M. \& Sonawane, A. Nanoparticle vaccines against infectious diseases. Front. Immunol. 9, 2224 (2018).

5. Kaba, S. A. et al. Self-assembling protein nanoparticles with built-in flagellin domains increases protective efficacy of a Plasmodium falciparum based vaccine. Vaccine 36, 906-914 (2018).

6. Boyaka, P. N. Inducing mucosal IgA: a challenge for vaccine adjuvants and delivery systems. J. Immunol. 199, 9-16 (2017).

7. Savelkoul, H. F., Ferro, V. A., Strioga, M. M. \& Schijns, V. E. Choice and design of adjuvants for parenteral and mucosal vaccines. Vaccines 3, 148-171 (2015).

8. Yusuf, H. \& Kett, V. Current prospects and future challenges for nasal vaccine delivery. Hum. Vaccines Immunother. 13, 34-45 (2017).

9. Kim, S. H. \& Jang, Y. S. The development of mucosal vaccines for both mucosal and systemic immune induction and the roles played by adjuvants. Clin. Exp. Vaccine Res. 6, 15-21 (2017).

10. Lambkin, R. et al. Strong local and systemic protective immunity induced in the ferret model by an intranasal virosome-formulated influenza subunit vaccine. Vaccine 22, 4390-4396 (2004).

11. Bozzuto, G. \& Molinari, A. Liposomes as nanomedical devices. Int. J. Nanomed. 10, 975-999 (2015).

12. Schwendener, R. A. Liposomes as vaccine delivery systems: a review of the recent advances. Ther. Adv. Vaccines 2, 159-182 (2014).

13. Agren, L. C., Ekman, L., Lowenadler, B., Nedrud, J. G. \& Lycke, N. Y. Adjuvanticity of the cholera toxin A1-based gene fusion protein, CTA1-DD, is critically dependent on the ADP-ribosyltransferase and Ig-binding activity. J. Immunol. 162, 2432-2440 (1999).

14. Eriksson, A. M., Schon, K. M. \& Lycke, N. Y. The cholera toxin-derived CTA1-DD vaccine adjuvant administered intranasally does not cause inflammation or accumulate in the nervous tissues. J. Immunol. 173, 3310-3319 (2004).

15. Lycke, N. \& Lebrero-Fernandez, C. ADP-ribosylating enterotoxins as vaccine adjuvants. Curr. Opin. Pharmacol. 41, 42-51 (2018).

16. Bernasconi, V. et al. Porous nanoparticles with self-adjuvanting M2e-fusion protein and recombinant hemagglutinin provide strong and broadly protective immunity against influenza virus infections. Front. Immunol. 9, 2060 (2018).

17. Agren, L., Lowenadler, B. \& Lycke, N. A novel concept in mucosal adjuvanticity: the CTA1-DD adjuvant is a B cell-targeted fusion protein that incorporates the enzymatically active cholera toxin A1 subunit. Immunol. Cell Biol. 76, 280-287 (1998).

18. Eliasson D. G., et al. M2e-tetramer-specific memory CD4 T cells are broadly protective against influenza infection. Mucosal Immunol. 11, 273-289 (2017).

19. Kolpe, A., Schepens, B., Fiers, W. \& Saelens, X. M2-based influenza vaccines: recent advances and clinical potential. Expert Rev. Vaccines 16, 123-136 (2017).

20. Deng, L., Cho, K. J., Fiers, W. \& Saelens, X. M2e-based universal influenza A vaccines. Vaccines 3, 105-136 (2015).

21. Eliasson, D. G. et al. CTA1-M2e-DD: a novel mucosal adjuvant targeted influenza vaccine. Vaccine 26, 1243-1252 (2008).
22. Kappler, J., White, J., Wegmann, D., Mustain, E. \& Marrack, P. Antigen presentation by la+ B cell hybridomas to H-2-restricted T cell hybridomas. Proc. Natl Acad. Sci. USA 79, 3604-3607 (1982)

23. Murphy, D. B. et al. A novel MHC class II epitope expressed in thymic medulla but not cortex. Nature 338, 765-768 (1989).

24. Avalos, A. M. \& Ploegh, H. L. Early BCR events and antigen capture, processing, and loading on MHC Class II on B Cells. Front. Immunol. 5, 92 (2014).

25. Ashhurst, A. S. et al. CXCR6-deficiency improves the control of pulmonary mycobacterium tuberculosis and influenza infection independent of T-Lymphocyte recruitment to the lungs. Front. Immunol. 10, 339 (2019).

26. Schild, G. C. et al. Antigenic variation in current human type $A$ influenza viruses: antigenic characteristics of the variants and their geographic distribution. Bull. World Health Organ. 48, 269-278 (1973).

27. Wein, A. N. et al. CXCR6 regulates localization of tissue-resident memory CD8 T cells to the airways. J. Exp. Med. 216, 2748-2762 (2019).

28. Eliasson, D. G. et al. A novel non-toxic combined CTA1-DD and ISCOMS adjuvant vector for effective mucosal immunization against influenza virus. Vaccine 29 , 3951-3961 (2011).

29. Norling, K., et al. Gel Phase 1,2-Distearoyl-sn-glycero-3-phosphocholine-based liposomes are superior to fluid phase liposomes at augmenting both antigen presentation on major histocompatibility complex Class II and costimulatory molecule display by dendritic cells in vitro. ACS Infect. Dis. 5, 1867-1878 (2019).

30. Nance, E. et al. Brain-penetrating nanoparticles improve paclitaxel efficacy in malignant glioma following local administration. ACS Nano 8, 10655-10664 (2014).

31. Oberoi, H. S., Yorgensen, Y. M., Morasse, A., Evans, J. T. \& Burkhart, D. J. PEG modified liposomes containing CRX-601 adjuvant in combination with methylglycol chitosan enhance the murine sublingual immune response to influenza vaccination. J. Control Release 223, 64-74 (2016).

32. Suk, J. S., Xu, Q., Kim, N., Hanes, J. \& Ensign, L. M. PEGylation as a strategy for improving nanoparticle-based drug and gene delivery. Adv. Drug Deliv. Rev. 99(Pt A), 28-51 (2016).

33. Ji, Z., Xie, Z., Zhang, Z., Gong, T. \& Sun, X. Engineering intravaginal vaccines to overcome mucosal and epithelial barriers. Biomaterials 128, 8-18 (2017).

34. Zhan, X., Tran, K. K. \& Shen, H. Effect of the poly(ethylene glycol) (PEG) density on the access and uptake of particles by antigen-presenting cells (APCs) after subcutaneous administration. Mol. Pharm. 9, 3442-3451 (2012).

35. Xu, Q. et al. Impact of surface polyethylene glycol (PEG) density on biodegradable nanoparticle transport in Mucus ex vivo and distribution in vivo. ACS Nano 9, 9217-9227 (2015).

36. Watson, D. S., Endsley, A. N. \& Huang, L. Design considerations for liposomal vaccines: influence of formulation parameters on antibody and cell-mediated immune responses to liposome associated antigens. Vaccine 30, 2256-2272 (2012).

37. Rincon-Restrepo, M. et al. Vaccine nanocarriers: coupling intracellular pathways and cellular biodistribution to control CD4 vs CD8 T cell responses. Biomaterials 132, 48-58 (2017).

38. Schlitzer, A., Zhang, W., Song, M., Ma, X. Recent advances in understanding dendritic cell development, classification, and phenotype. F1000Res. 7, (2018).

39. Flores-Langarica, A. et al. Intestinal CD103(+)CD11b(+) CDC2 conventional dendritic cells are required for primary $\mathrm{CD} 4(+) \mathrm{T}$ and $\mathrm{B}$ cell responses to soluble flagellin. Front. Immunol. 9, 2409 (2018).

40. Helft, J. et al. Cross-presenting CD103+ dendritic cells are protected from influenza virus infection. J. Clin. Investig. 122, 4037-4047 (2012)

41. Radford, K. J. \& Caminschi, I. New generation of dendritic cell vaccines. Hum. Vaccine Immunother. 9, 259-264 (2013).

42. Lee, H. et al. Phenotype and function of nasal dendritic cells. Mucosal Immunol. 8, 1083-1098 (2015).

43. Neirynck, S. et al. A universal influenza A vaccine based on the extracellular domain of the M2 protein. Nat. Med. 5, 1157-1163 (1999).

44. De Filette, M. et al. Universal influenza A vaccine: optimization of M2-based constructs. Virology 337, 149-161 (2005).

45. Leung, H. C. et al. An H5N1-based matrix protein 2 ectodomain tetrameric peptide vaccine provides cross-protection against lethal infection with H7N9 influenza virus. Emerg. Microbes Infect. 4, e22 (2015).

46. Chwalek, M., Lalun, N., Bobichon, H., Ple, K. \& Voutquenne-Nazabadioko, L. Structure-activity relationships of some hederagenin diglycosides: haemolysis, cytotoxicity and apoptosis induction. Biochim. Biophys. Acta 1760, 1418-1427 (2006).

47. Spangler, B. D. Structure and function of cholera toxin and the related Escherichia coli heat-labile enterotoxin. Microbiol. Rev. 56, 622-647 (1992). 